\title{
Pharmacogenomics of intracellular methotrexate polyglutamates in patients' leukemia cells in vivo
}

\author{
Elixabet Lopez-Lopez, ${ }^{1,2}$ Robert J. Autry, ${ }^{1,2,3}$ Colton Smith, ${ }^{1,2}$ Wenjian Yang, ${ }^{1,2}$ Steven W. Paugh, ${ }^{1,2}$ John C. Panetta, ${ }^{2}$ \\ Kristine R. Crews, ${ }^{1,2}$ Erik J. Bonten, ${ }^{1,2}$ Brandon Smart, ${ }^{2}$ Deqing Pei, ${ }^{4}$ J. Robert McCorkle, ${ }^{1,2}$ Barthelemy Diouf, ${ }^{1,2}$ \\ Kathryn G. Roberts, ${ }^{1,5}$ Lei Shi, ${ }^{4}$ Stanley Pounds, ${ }^{4}$ Cheng Cheng, ${ }^{4}$ Charles G. Mullighan, ${ }^{1,5}$ Ching-Hon Pui, $, 1,5,6$ \\ Mary V. Relling, ${ }^{1,2,3}$ and William E. Evans ${ }^{1,2,3}$ \\ ${ }^{1}$ Hematological Malignancies Program, and '2Department of Pharmaceutical Sciences, St. Jude Children's Research Hospital, Memphis, Tennessee, USA. ${ }^{3}$ College of Graduate Health Sciences, \\ University of Tennessee Health Science Center, Memphis, Tennessee, USA. ${ }^{4}$ Department of Biostatistics, ${ }^{5}$ Department of Pathology, and ${ }^{6}$ Department of Oncology, St. Jude Children's Research Hospital, \\ Memphis, Tennessee, USA.
}

\begin{abstract}
BACKCROUND. Interpatient differences in the accumulation of methotrexate's active polyglutamylated metabolites (MTXPGs) in leukemia cells influence its antileukemic effects.
\end{abstract}

\begin{abstract}
METHODS. To identify genomic and epigenomic and patient variables determining the intracellular accumulation of MTXPGs, we measured intracellular MTXPC levels in acute lymphoblastic leukemia (ALL) cells from 388 newly diagnosed patients after in vivo high-dose methotrexate (HDMTX) $\left(1 \mathrm{~g} / \mathrm{m}^{2}\right)$ treatment, defined ALL subtypes, and assessed genomic and epigenomic variants influencing folate pathway genes (mRNA, miRNA, copy number alterations [CNAs], SNPs, single nucleotide variants [SNVs], CpG methylation).

RESULTS. We documented greater than 100-fold differences in MTXPG levels, which influenced its antileukemic effects ( $P$ $=4 \times 10^{-5}$ ). Three ALL subtypes had lower MTXPC levels (T cell ALL [T-ALL] and B cell ALL [B-ALL] with the TCF3-PBX1 or ETV6-RUNX1 fusions), and 2 subtypes had higher MTXPC levels (hyperdiploid and BCR-ABL like). The folate pathway genes SLC19A1, ABCC1, ABCC4, FPCS, and MTHFD1 significantly influenced intracellular MTXPC levels $\left(P=2.9 \times 10^{-3}\right.$ to $\left.3.7 \times 10^{-8}\right) . A$ multivariable model including the ALL subtype $\left(P=1.1 \times 10^{-14}\right)$, the $S L C 19 A 1 /(A B C C 1+A B C C 4)$ transporter ratio $\left(P=3.6 \times 10^{-4}\right)$, the MTX infusion time $\left(P=1.5 \times 10^{-3}\right)$, FPCS mRNA expression $\left(P=2.1 \times 10^{-3}\right)$, and MTX systemic clearance $\left(P=4.4 \times 10^{-2}\right)$ explained $42 \%$ of the variation in MTXPG accumulation $\left(P=1.1 \times 10^{-38}\right)$. Model simulations indicated that a longer infusion time (24 h vs. $4 \mathrm{~h}$ ) was superior in achieving higher intracellular MTXPG levels across all subtypes if ALL.
\end{abstract}

CONCLUSIONS. These findings provide insights into mechanisms underlying interpatient differences in intracellular accumulation of MTXPG in leukemia cells and its antileukemic effects

FUNDING. THE National Cancer Institute (NCI) and the Institute of General Medical Sciences of the NIH, the Basque Government Programa Posdoctoral de Perfeccionamiento de Personal Investigador doctor, and the American Lebanese Syrian Associated Charities (ALSAC).

\section{Introduction}

Acute lymphoblastic leukemia (ALL) is a common disseminated malignancy that is cured by combination chemotherapy in approximately $90 \%$ of children and $70 \%$ of adults (1-3). Achieving sufficient concentrations of active drug in cancer cells is condicio sine qua non for inhibition of intracellular targets, and differences in intracellular drug accumulation contribute to differences in treatment response (4).

High-dose methotrexate (HDMTX) is a critical component of curative treatment of children with ALL, and the response to

Conflict of interest: The authors have declared that no conflict of interest exists. Copyright: $\odot$ 2020, American Society for Clinical Investigation.

Submitted: June 1, 2020; Accepted: August 20, 2020; Published: November 9, 2020

Reference information: J Clin Invest. 2020;130(12):6600-6615.

https://doi.org/10.1172/JCl140797.
HDMTX is influenced by the extracellular concentration of methotrexate (MTX) in patients $(5,6)$, which influences the intracellular accumulation of active methotrexate polyglutamate metabolites (MTXPGs) (7, 8). The leukemia cells of patients with T-lineage ALL are known to accumulate lower MTXPG levels than patients with B-lineage ALL, largely because of lower expression of folylpolyglutamate synthetase (FPGS). It has also been shown that ALL cells with the ETV6-RUNX1 chromosomal fusion or the TCF3-PBX1 fusion accumulate lower MTXPG levels, whereas patients whose ALL cells are hyperdiploid ( $>50$ chromosomes) accumulate significantly higher MTXPG levels (7-9). More recently, new molecular ALL subtypes have been discovered, including the $B C R-A B L$-like ALL subtype $(10,11)$ and the DUX4/ERG-deregulated subtype (12), but it is not known how well they accumulate intracellular MTXPG.

We have previously shown in a prospective, randomized clinical trial that HDMTX $\left(1 \mathrm{~g} / \mathrm{m}^{2}\right)$ infused over 24 hours achieves 


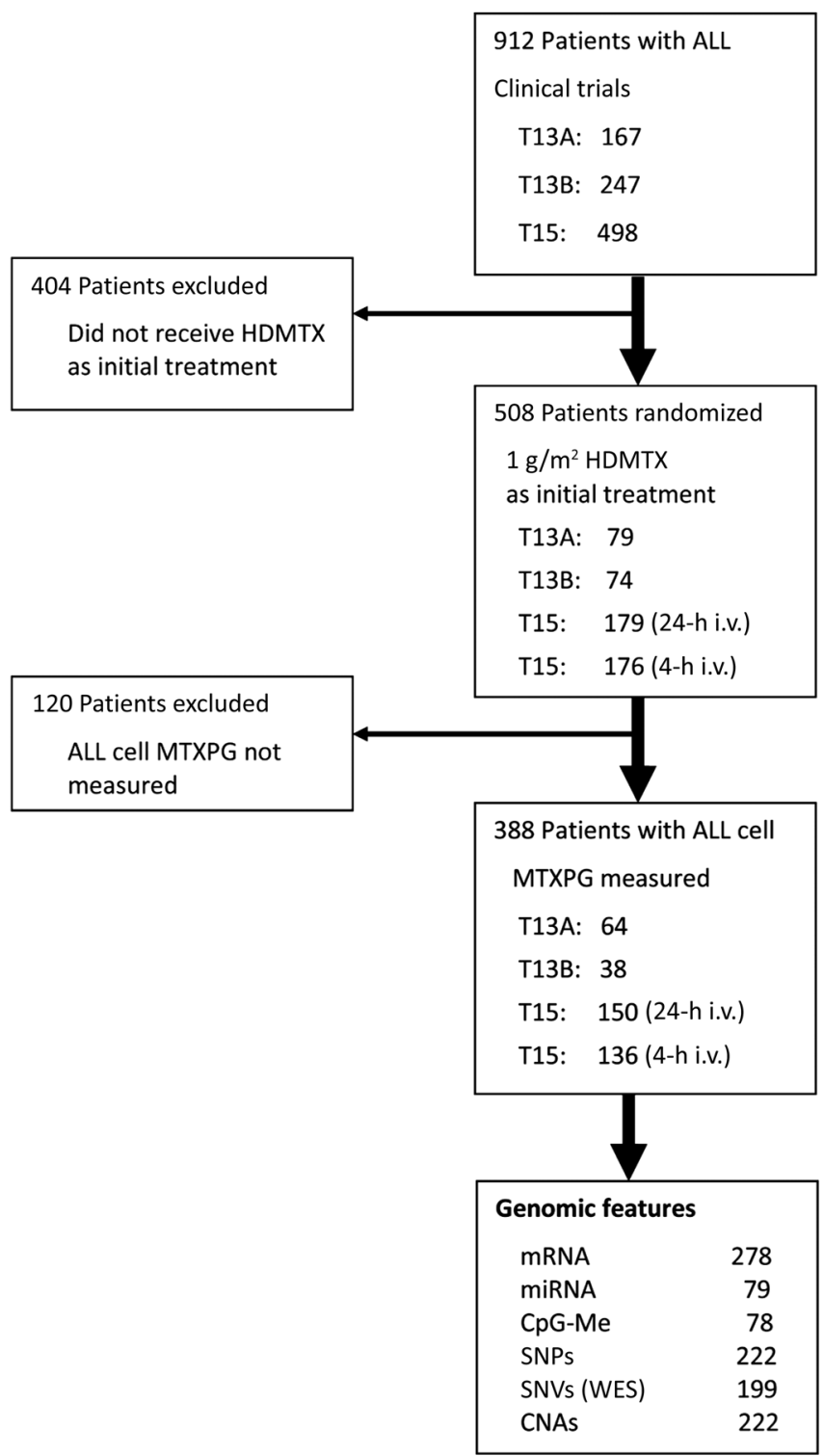

higher intracellular levels of active MTXPG in primary ALL cells in vivo when compared with a 4-hour infusion (13), but the impact of a longer infusion time has not been established for individual molecular subtypes of ALL.

In the current study, we investigated all major subtypes of ALL, including more recently identified subtypes, to assess potential determinants of MTXPG intracellular accumulation, including HDMTX infusion time, genomic and epigenomic variants linked to folate pathway genes (i.e., mRNA and miRNA expression, germline SNPs, coding variants by whole-exome sequencing, CpG methylation and copy number alterations [CNAs]) and MTX systemic clearance. We show that a multivariable model with ALL molecular subtype, the folate transporter ratio (mRNA expression of $S L C 19 A 1 /(A B C C 1+A B C C 4))$, the MTX infusion time, FPGS expression, and MTX systemic clearance explains $42 \%$ of the variability in intracellular MTXPG accumulation in vivo $\left(P=1.14 \times 10^{-38}\right)$. We observed that greater antileukemic effects of HDMTX occurred in patients whose ALL cells accumulated higher intracellular MTXPG levels.
Figure 1. CONSORT diagram depicting patient enrollment. All patients enrolled in the T15 protocol were randomized to receive HDMTX as either a 4-hour or 24-hour i.v. infusion. All patients enrolled in the T13A and T13B protocols received HTMTX as a 24-hour i.v. infusion. T13A, total XIIIA treatment protocol (19); T13B, total XIIIB treatment protocol (20); T15, total XV treatment protocol (NCT00137111; ref. 18). mRNA, mRNA expression; miRNA, miRNA expression; CpG-Me, CpG methylation. mRNA and miRNA expression, CpG methylation, SNPs, SNVs, and CNAs were analyzed in ALL cells, as described in Methods.

\section{Results}

MTXPG accumulation in patients' leukemia cells. The accumulation of total MTXPG (1-7 glutamate moieties) in leukemia cells 42-44 hours after in vivo HDMTX treatment differed widely among the 388 pediatric patients (Figure 1) with newly diagnosed ALL ( $>100$ fold based on first percentile and 99th percentile values; coefficient of variation $[\mathrm{CV}]=119 \%$ ) (Figure $2 \mathrm{~A}$ ), and differed by ALL molecular subtype (Figure 2B).

The intracellular amount of long-chain MTXPG (4-7 glutamate moieties) also varied widely (Supplemental Figure 1A; supplemental material available online with this article; https://doi. org/10.1172/JCI140797DS1), differed by ALL subtype (Supplemental Figure $1 \mathrm{~B})$, and was highly correlated with the values for total MTXPG $\left(P=1.36 \times 10^{-224}\right)$ (Supplemental Figure 1C). MTXPGs with 3-5 glutamates were the most abundant forms in leukemia cells (Supplemental Figure 1D). Given the high correlation between total and long-chain MTXPGs, only the results for total MTXPG levels are presented in this study.

In a multivariable regression analysis, ALL lineage and molecular subtype $\left(P=7.29 \times 10^{-33}\right)$, MTX infusion time $\left(P=5.31 \times 10^{-4}\right)$, and MTX systemic clearance $\left(P=4.0 \times 10^{-3}\right)$ were each significantly related to intracellular MTXPG accumulation (Table 1). MTXPG accumulation was not associated with the demographic covariates of age, sex, or ancestry.

Patients randomized to receive a 24-hour infusion had higher accumulation of total MTXPG than did patients who received a 4-hour infusion, as previously reported (13) (Supplemental Figure 2). Patients with higher MTX systemic clearance, and thus lower extracellular blood concentrations of MTX, had lower intracellular MTXPG accumulation $\left(P=8.5 \times 10^{-3}\right)$ (Supplemental Figure 3).

As depicted in Figure 2B, patients with the $B C R-A B L$-like ALL subtype (with or without CRLF2 rearrangements) $(10,11)$ or hyperdiploid ALL $\left(P=2.52 \times 10^{-2}\right.$ and $2.65 \times 10^{-3}$, respectively, when compared with the B-other subtype, defined as B-ALL without any of the genomic changes that define the other 9 subtypes) had the highest average MTXPG accumulation, whereas patients with T cell ALL (T-ALL) $\left(P=3.36 \times 10^{-12}\right)$, or B-lineage ALL with the TCF3-PBX fusion $\left(P=4.63 \times 10^{-7}\right)$, or the ETV6-RUNX1 fusion $\left(P=8.14 \times 10^{-5}\right)$ had lower MTXPG accumulation.

Antileukemic effects related to MTXPG accumulation in leukemia. As depicted in Figure 2C, the decrease in the leukemia cell count over a 3-day period following single-agent HDMTX treatment, adjusted for the effect of baseline ALL cell counts (see Methods), was significantly associated with the in vivo level of MTXPG accumulation in leukemia cells $\left(3.62 \times 10^{-5} ; r=-0.251\right)$. The ALL subtypes for each patient depicted in Figure $2 \mathrm{C}$ are provided in Supplemental Figure 4. Similarly, as depicted in Figure 2D, patients who 

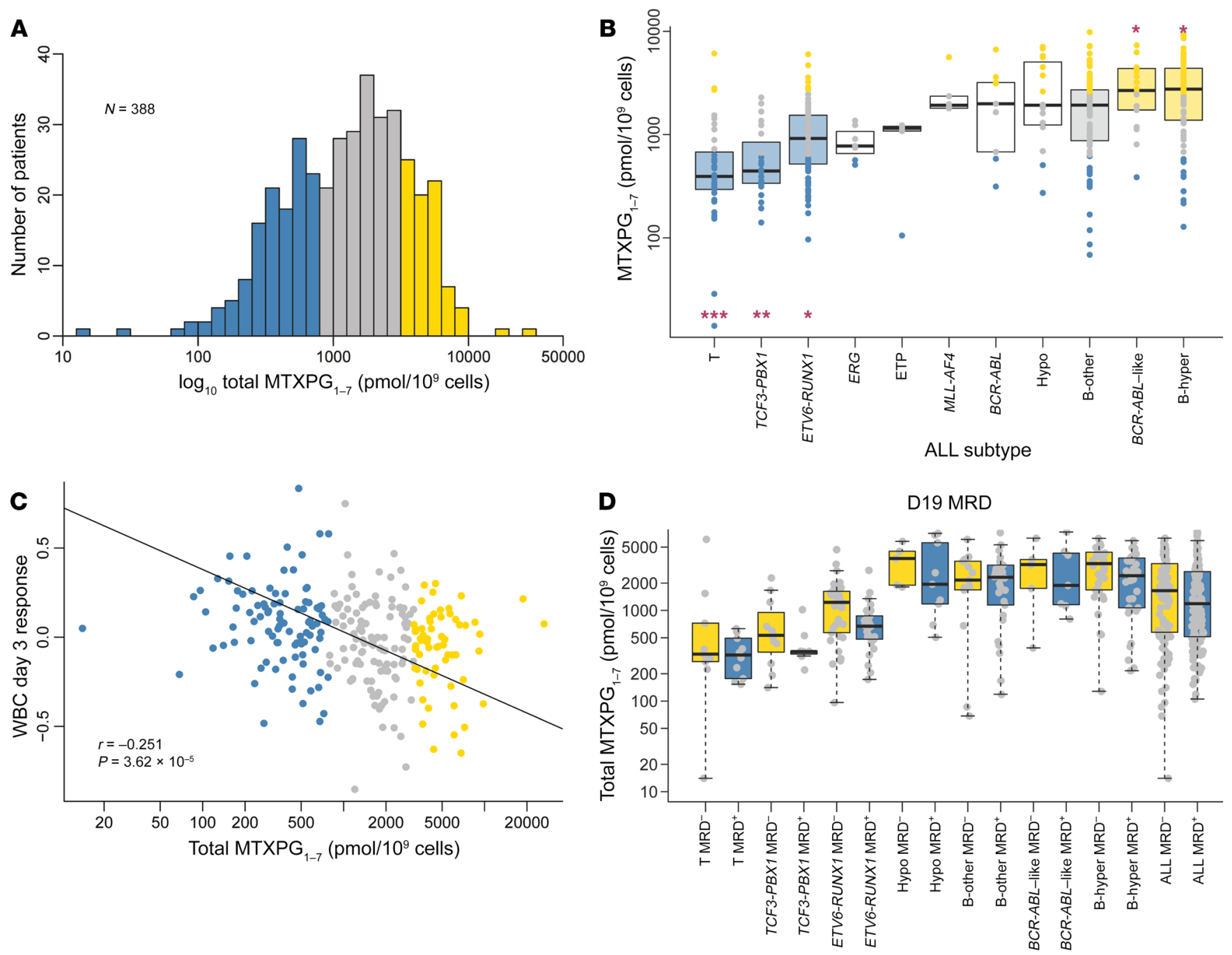

Figure 2. Total intracellular MTXPG in $_{1-7}$ bone marrow leukemia cells from 388 newly diagnosed patients with ALL, measured 42 hours after in vivo

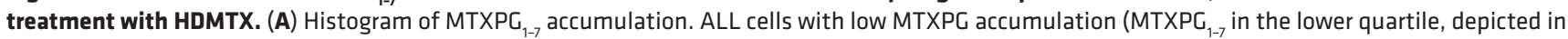
blue) ranged from 14.1-568.3 pmol/10 $10^{9}$ cells; ALL cells with high MTXPG accumulation (upper quartile, in gold) had MTXPG levels of 2724-26,479 pmol/109 cells. MTXPG concentrations in the 25th-75th percentile range are depicted in gray. (B) MTXPG ${ }_{1-7}$ accumulation for each major subtype of ALL, ordered from lowest to highest mean value. Each box depicts the 25th-75th percentiles, with the horizontal line indicating the median. Boxes shown in blue represent subtypes with $\mathrm{MTXPG}_{1-7}$ accumulation significantly lower $(P<0.05)$ than the B-other subgroup (in gray), whereas the boxes depicted in gold represent subtypes with $\mathrm{MTXPG}_{1-7}$ accumulation significantly higher. ${ }^{*} P<0.05,{ }^{* *} P<1 \times 10^{-5}$, and ${ }^{* *} P<1 \times 10^{-10}$, by $t$ test. Each data point represents a patient's ALL sample: samples from patients with $\mathrm{MTXPG}_{1-7}$ in the lower quartile are depicted in blue; those in the upper quartile in gold, and those in between in gray. (C) Relation between MTXPG accumulation in ALL cells and the decrease in leukemia cell count over 3 days following single-agent HDMTX treatment, adjusted for the effect of baseline ALL cell counts. The colors correspond to those in panel $\mathbf{B}$. The line depicts the best-fit linear model $(r=-0.251, P=4 \times$ $10^{-5}$, Pearson's correlation). (D) MTXPG concentrations in patients who were MRD- $(<0.01 \%)$ in bone marrow on day 19 (D19) of combination chemotherapy (gold boxes) versus patients who were $\mathrm{MRD}^{+}$(blue boxes) for each ALL subtype, with at least 10 patients, and for the entire cohort (ALL MRD- and ALL MRD'). The MTXPG concentration was significantly higher in patients who were MRD- $(P=0.043$, with subtype as a covariate; $P=0.040$, within only B-lineage ALL, data not shown, F test). Hypo, hypodiploid ALL; B-hyper, B-hyperdiploid ALL.

had residual leukemia detectable in bone marrow after 19 days of combination chemotherapy with HDMTX prednisone, vincristine, asparaginase, and daunorubicin had significantly lower MTXPG levels in their ALL cells after initial HDMTX treatment $(P=0.043$ with subtype as a covariate, $P=0.040$ within only B-lineage ALL). Inclusion of the mRNA expression of DHFR $(P=0.11)$ or TYMS $(P=0.53)$ did not significantly improve the model for ALL response to HDMTX treatment (data not shown).

Genomic and epigenomic features associated with MTXPG accumulation. Because MTX is a folic acid analog that is transported and metabolized by the same proteins as endogenous folates, we focused on the expression of 47 folate pathway genes (Supplemental Table 1). Assessed as a continuous variable by linear regression (Figure 3), the mRNA expression of 4 of these genes (FPGS, SLC19A1, MTHFD1, and ABCC1) was significantly related to the level of MTXPG accumulation in ALL cells, after correcting for multiple testing $(P<0.001)$. The level of expression of these 4 genes alone or with $A B C C 4$ (the only other folate transporter significantly related to MTXPG, $P=0.031$; Figure 3I) discriminated between patients with high (upper quartile) versus low (lowest 


\section{Table 1. Nongenetic factors influencing MTXPG accumulation with ALL subtype as a covariate}

\begin{tabular}{lccc} 
Covariate & Estimate & SE & $P$ value \\
Multivariable regression model & & & \\
Sex (male vs. female) & -0.0034 & 0.041 & 0.935 \\
Age (yr) & 0.0023 & 0.0048 & 0.63 \\
\hline Ancestry & - & - & 0.37 \\
\hline MTX systemic clearance & -0.123 & 0.042 & 0.0040 \\
\hline MTX infusion time (4 h vs. 24 h) & -0.152 & 0.043 & $5.31 \times 10^{-4}$ \\
\hline ALL subtype & - & - & $7.29 \times 10^{-33}$ \\
\hline Multivariable regression model after stepwise variable selection & \\
\hline MTX systemic clearance & -0.252 & 0.0742 & $7.87 \times 10^{-4}$ \\
\hline MTX infusion time (4 h vs. 24 h) & -0.191 & 0.0475 & $7.22 \times 10^{-5}$ \\
\hline ALL subtype (vs. B-other) & & & \\
\hline T & -0.6204 & 0.0866 & $4.45 \times 10^{-12}$ \\
\hline TCF3-PBX1 & -0.4287 & 0.0993 & $2.27 \times 10^{-5}$ \\
\hline ETV6-RUNX1 & -0.1658 & 0.0717 & $2.14 \times 10^{-2}$ \\
\hline ERG & -0.1833 & 0.1522 & $2.30 \times 10^{-1}$ \\
\hline ETP & -0.2706 & 0.1773 & $1.28 \times 10^{-1}$ \\
\hline MLL-AF4 & 0.1347 & 0.1760 & 0.445 \\
\hline BCR-ABL & -0.0679 & 0.1624 & $6.76 \times 10^{-1}$ \\
\hline Hypodiploid & 0.2352 & 0.1309 & $7.36 \times 10^{-2}$ \\
\hline BCR-ABL-like & 0.2855 & 0.1034 & $6.15 \times 10^{-3}$ \\
\hline Hyperdiploid & 0.3107 & 0.0731 & $2.97 \times 10^{-5}$ \\
\hline Overall significance & & & $4.88 \times 10^{-34}$ \\
\hline
\end{tabular}

Estimate, estimated coefficient; SE, standard error of the coefficient estimate. For sex, "female" was used as the reference; for MTX infusion time, 24 hours was used as the reference (thus negative values indicate that a 4-hour infusion time was inferior to a 24 -hour infusion time).

quartile) MTXPG accumulation (clustering $P=2.60 \times 10^{-9}$; Figure 4), independent of ALL subtype. In a subset of these patients $(n=22)$, we measured the expression of these 5 folate pathway genes 42 hours after HDMTX treatment and detected no significant change from the pretreatment mean value for any gene ( $P=0.016-0.87$, data not shown). The absence of an association of MTXPG with other ATP-binding cassette (ABC) transporters is depicted in Supplemental Figure 5.

MTXPG accumulation was positively correlated with the expression of FPGS $\left(P=3.7 \times 10^{-8}\right.$; Figure $\left.3 \mathrm{~A}\right)$, and FPGS expression was significantly lower in T-ALL, ETV6-RUNX1, and hyperdiploid B-lineage ALL cells (Figure 3B). The relation between MTXPG and expression of the other 4 folate pathway genes (SLC19A1, MTHFD1, ABCC1, ABCC4) and their subtype differences in expression are shown in Figure 3, C-J.

Because the accumulation of MTXPG in ALL cells is likely influenced by the net effect of the ratio of mRNA expression of the MTX influx transporter SLC19A1 to the expression of the efflux transporters $A B C C 1$ and $A B C C 4$, we assessed the relation between intracellular MTXPG and the folate transporter ratio of $S L C 19 A 1 /(A B C C 1+A B C C 4)$. As depicted in Figure 5A, the intracellular accumulation of MTXPG was significantly related to the ratio of the influx/efflux transporters $\left(P=1.37 \times 10^{-11}\right)$. The strongest association between MTXPG and the influx/efflux trans- porter ratio was obtained with the transporter ratio of SLC19A1/ $(A B C C 1+A B C C 4)$, compared with SLC19A1/ABCC1 $(P=5.2 \times$ $\left.10^{-11}\right)$ or $S L C 19 A 1 / A B C C 4\left(P=7 \times 10^{-8}\right)$. This transporter ratio was significantly lower in the 3 ALL subtypes with low MTXPG accumulation compared with the 2 subtypes with high MTXPG accumulation $\left(P=1.77 \times 10^{-11}\right.$; Figure $\left.5 \mathrm{~B}\right)$.

To further assess the importance of genomic and epigenomic variants in folate pathway genes for their relation to MTXPG accumulation in ALL cells, we used the truncated aggregation of $P$ values (TAP) statistic (see Methods) to interrogate 6 types of genomic and epigenomic variables (i.e., mRNA and miRNA expression, germline SNPs, coding variants by whole-exome sequencing [WES], $\mathrm{CpG}$ methylation, and CNAs) in leukemia cells, aggregated to each folate pathway gene. Table 2 summarizes the TAP statistic for each folate pathway gene with a $P$ value of less than 0.05 , highlighting the 10 genes that achieved statistical significance after correcting for multiple testing $(P<0.005)$. The top folate pathway gene in the TAP analysis was GART (ranked seventh by mRNA and ranked first after other genomic features were interrogated; Supplemental Figure 6, A-L). GART encodes a trifunctional enzyme (also known as glycinamide ribonucleotide formyltransferase, glycinamide ribonucleotide synthetase, and aminoimidazole ribonucleotide synthetase) involved in the conversion of phosphoribosyl pyrophosphate to inosine monophosphate in the de novo purine synthesis pathway, using 10-formyl tetrahydrofolate (THF) as a substrate. THF is produced from 5,10 methenyl-THF by MTHFD1, a folate pathway gene significantly related to MTXPG accumulation in ALL cells (Figure 4).

Multiple treatment, pharmacokinetic, and genomic variables influence MTXPG intracellular accumulation in leukemia cells in vivo. In a multivariable analysis (Figure 5C, Table 3, and Supplemental Tables 2 and 3), we found that intracellular MTXPG accumulation was significantly related to the ALL molecular subtype $\left(P=1.1 \times 10^{-14}\right)$, the $S L C 19 A 1 /(A B C C 1+A B C C 4)$ transporter ratio $\left(P=3.6 \times 10^{-4}\right)$, the MTX infusion time $\left(P=1.5 \times 10^{-3}\right), F P G S$ mRNA expression $(P=$ $\left.2.1 \times 10^{-3}\right)$, and MTX systemic clearance $\left(P=4.4 \times 10^{-2}\right)$, collectively explaining $42 \%$ of the variation in MTXPG accumulation across the cohort $\left(P=1.14 \times 10^{-38}\right)$. The analysis also showed that there were 4 cis $\mathrm{CpG}$ methylation sites significantly related to both MTXPG and inversely related to the mRNA expression level of FPGS (cg24910675 $\left.P=5.6 \times 10^{-6}, \operatorname{cg} 05050341 P=1.2 \times 10^{-5}\right), S L C 19 A 1(\operatorname{cg} 07658590 P=$ $\left.5.2 \times 10^{-5}\right)$, and $A B C C 4\left(\operatorname{cg} 15868151, P=7.1 \times 10^{-4}\right)$, but none of these $\mathrm{CpG}$ sites remained significant in a multivariable model explaining MTXPG variation, when the level of mRNA expression of the corresponding gene was also included in the model.

Expression of FPGS and SLC19A1 correlates with expression of PBX1 and the NOTCH 2 membrane receptor. To identify genes that alter the expression of FPGS, we examined the intersection of 3 analyses: (a) genes significantly $\left(P<1 \times 10^{-6}\right)$ associated with FPGS expression, (b) genes significantly $\left(P<1 \times 10^{-6}\right)$ associated with MTXPG accumulation, and (c) genes whose promoters have transcriptional binding sites for at least 1 of the trans-acting factors altered in subtypes of ALL (PBX1, RUNX1, as compiled in the Molecular Signatures Database [MSigDB]). The top-ranked gene in this analysis was NOTCH2 (Figure 6, A and B), which encodes a transmembrane receptor involved in intercellular and intracellular signaling and transcriptional activation of target genes (14). 
A
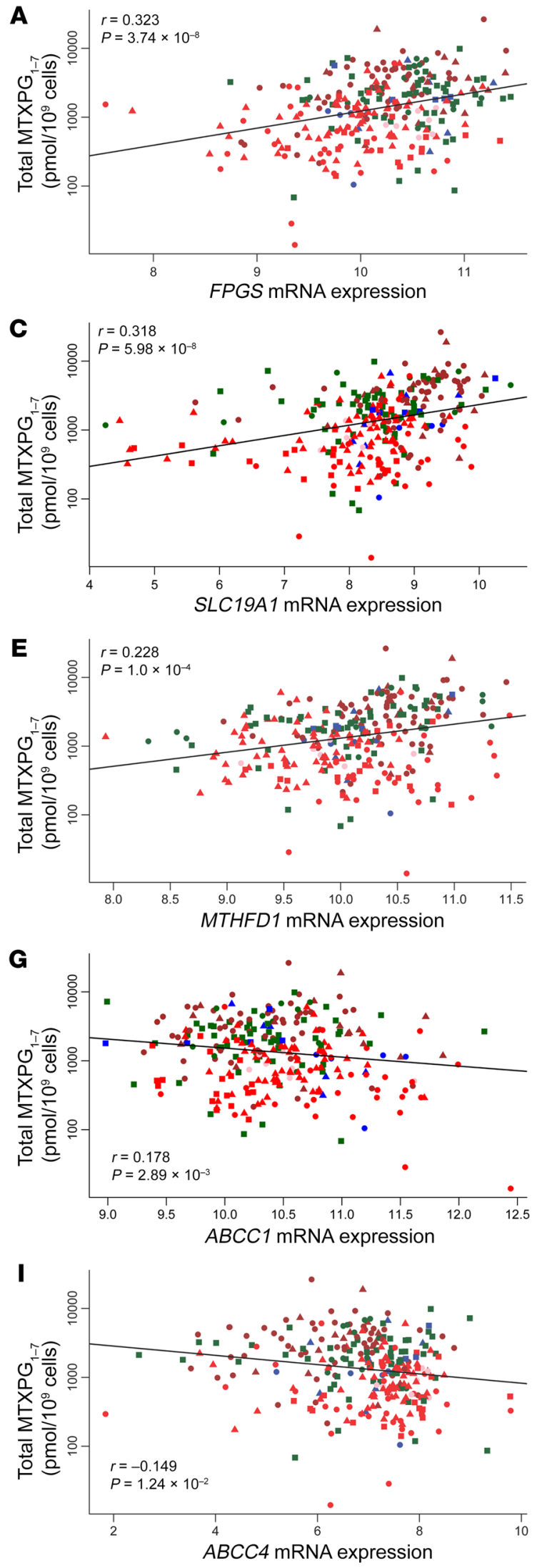

- TCF3-PBX1

ETV6-RUNX1

ERG

- ETP

MLL-AF4

- BCR-ABL

- Hypo

- B-other

BCR-ABL-like

- B-hyper
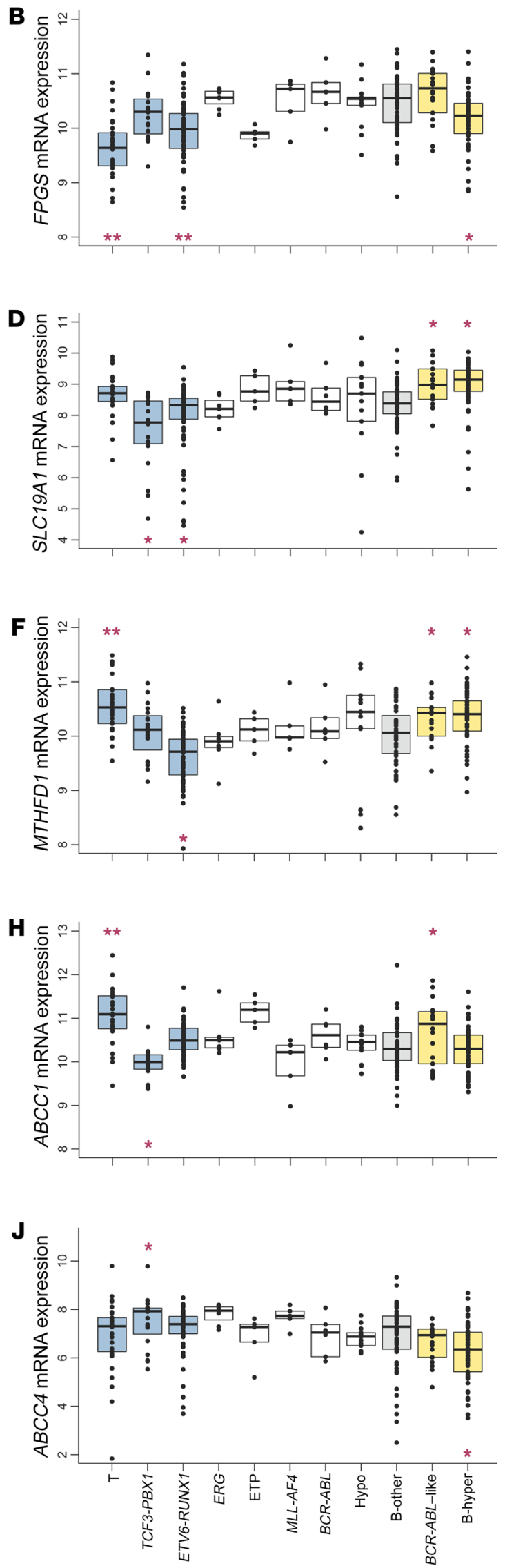

ALL subtype 
Figure 3. Relation between the expression of folate pathway genes and MTXPG accumulation in ALL cells, in vivo, and subtype differences in expression of folate genes in primary ALL cells. (A) mRNA expression of FPGS in primary ALL cells and in vivo accumulation of MTXPG after treatment with high-dose MTX. Each major subtype is depicted with a different symbol, as indicated in the symbol key. (B) Expression of FPGS mRNA in primary ALL cells across the major subtypes of ALL (blue represents subtypes with significantly lower MTXPG accumulation versus B-other [in gray], and gold represents subtypes with significantly higher MTXPC accumulation). (C-J) Data for mRNA expression of SLC19A1, MTHFD1, $A B C C 1$, and $A B C C 4$, respectively, as described for FPGS mRNA expression in $\mathbf{A}$ and $\mathbf{B}$. Line depicts the best-fit linear model, Pearson's correlation, and $P$ values. ${ }^{*} P<0.05$ and ${ }^{* *} P<1 \times 10^{-5}$, by $t$ test.

We found that the level of expression of FPGS was inversely correlated with the expression of NOTCH2 in primary ALL cells $\left(P=3.92 \times 10^{-9}\right.$; Figure 6A) and that NOTCH2 was expressed at a higher level in the 3 ALL subtypes (T-, TCF3-PBX1, and ETV6RUNX1) with the lowest MTXPG accumulation (Figure 6B). Leukemia cell expression of the folate uptake transporter SLC19A1 was also inversely correlated with NOTCH2 expression $(P=5.53$ $\times 10^{-4}$; Figure $6 \mathrm{C}$ ). NOTCH2 expression was significantly correlated with $P B X 1$ expression $\left(P=9.21 \times 10^{-10}\right.$; Figure $\left.6 \mathrm{D}\right)$, and $P B X 1$ expression was markedly higher in TCF3-PBX1 ALL (Figure 6E), the B-lineage ALL subtype with the highest levels of NOTCH2 expression (Figure 6B) and lowest MTXPG levels. Correspondingly, we noted that MTXPG accumulation was inversely correlated with $P B X 1$ expression in primary ALL cells $(r=-0.206, P=5.43 \times$ $10^{-4}$; data not shown).

Recapitulation of low MTXPG levels by overexpressing TF3-PBX1 or manipulating FPGS or SLC19A1 in human ALL cell lines. Because primary ALL cells with the TCF3-PBX1 fusion had the lowest intracellular MTXPG levels among all B-lineage ALL subtypes (Figure $2 \mathrm{~B})$, we overexpressed the TCF3-PBX1b fusion in the human $\mathrm{REH}$ B-lineage ALL cell line (Figure 7, A and B). This led to a decrease of approximately $40 \%$ in FPGS and SLC19A1 expression (Figure 7A), recapitulating what we observed in primary ALL cells. FPGS had a strong PBX1-binding site located 675 bp upstream of the transcription start site (TSS) $\left(P<1 \times 10^{-6}\right)(15)$, whereas SLC19A1 had a weaker PBX1-binding site located 2949 bp upstream of the TSS $\left(P<1 \times 10^{-3}\right)$. Overexpression of TCF3-PBX1 also caused a significant increase in NOTCH2 expression (Figure 7A), consistent with the known PBX1-binding site (per MsigDB) in the $5^{\prime}$ region of $\mathrm{NOTCH} 2$ (located 1655 bp upstream of the TSS, $P<1 \times 10^{-5}$ ). Further, overexpression of TCF3-PBX1 led to a 33\% decrease in total intracellular MTXPG $\left(P<1.0 \times 10^{-6}\right)$, with a $37.8 \%$ decrease in long-chain MTXPGs and a $20.3 \%$ decrease in short-chain MTXPGs (Figure 7A). This is consistent with the lower MTXPG levels and lower SLC19A1 expression observed in primary ALL cells with the TFC3-PBX1 fusion (Figures $2 \mathrm{~B}$ and Figure 4D) and with the significant inverse correlation between NOTCH2 and SLC19A1 or FPGS in primary ALL cells (Figure 6, A and C). Knockdown ( 80\%) of NOTCH2 in REH or 697 ALL cells led to an increase of approximately $40 \%$ in SLC19A1 expression, but no change in FPGS expression (data not shown). The hypothetical relation between expression of TCF3-PBX1 and expression of NOTCH2, FPGS, and SLC19A1 is depicted in Figure 7C.

As depicted in Figure 8A, overexpression of SLC19A1 in the human 697 ALL cell line led to a significant increase in total, short-chain, and long-chain MTXPGs $\left(P<1.0 \times 10^{-6}\right)$. Furthermore, knockdown of SLC19A1 expression in human 697, REH, and NALM6 ALL cell lines led to a significant reduction in total, shortchain, and long-chain MTXPGs $\left(P<1.0 \times 10^{-6}\right.$; Figure $\left.8 \mathrm{~B}\right)$. Likewise, knockdown of FPGS expression led to a significant decrease in total and long-chain MTXPGs in human 697 and NALM6 ALL cell lines $\left(P<1.0 \times 10^{-6}\right.$; Figure $\left.8 \mathrm{~B}\right)$.

The impact of HDMTX infusion time is evident across all levels of transporter ratios and FPGS expression. As depicted in Figure 9, model simulations indicated that $\operatorname{HDMTX}\left(1 \mathrm{~g} / \mathrm{m}^{2}\right)$ infused over 24 hours resulted in significantly higher intracellular accumulation of MTXPG than did HDMTX infused over 4 hours, across the entire spectrum of folate transporter ratios and FPGS expression. Representative simulations of intracellular accumulation of MTXPG are depicted in Supplemental Figure 7.

\section{Discussion}

Interpatient differences in the intracellular accumulation of MTXPG influence the anti-leukemic effects of MTX $(8,13,16$, 17). Indeed, in the current study, we documented a significant relationship between the level of intracellular MTXPG and the decrease in circulating leukemia cells 3 days after single-agent treatment with HDMTX (Figure 2C). As a second measure of antileukemic effects, we also documented significantly higher MTXPG levels in the leukemia cells of patients who were subsequently negative for minimal residual disease (MRD) $(<0.01 \%)$ in their bone marrow after 19 days of remission induction chemotherapy (Figure 2D).

We also further documented the superiority of 24-hour infusions of HDMTX compared with 4-hour infusions for achieving maximum intracellular MTXPG levels across all 11 subtypes of ALL. We also documented the influence of MTX systemic clearance, as patients with higher MTX clearance were found to have lower extracellular MTX concentrations and hence lower intracellular MTXPG levels, consistent with previous studies $(5-7,13)$. For these reasons, we included each patient's HDMTX infusion time and MTX systemic clearance as covariates for all genomic and epigenomic analyses.

The current studies provide insights into the determinants of interpatient differences in MTXPG levels in leukemia cells ( $>100$ fold) following identical i.v. treatment (Figure 2A) as a basis for more precise dosing of HDMTX. We identified genomic (chromosomal translocations, expression of folate pathway genes), treatment (infusion time), and pharmacokinetic characteristics (MTX systemic clearance) that significantly influenced MTXPG accumulation in leukemia cells following in vivo treatment with i.v. HDMTX. A multivariable model including the ALL subtype, the folate influx/efflux transporter expression ratio, the HDMTX infusion time, FPGS expression, and MTX systemic clearance explained $42 \%$ of the interpatient variation in MTXPG accumulation across the entire population $\left(P=1.14 \times 10^{-38}\right.$; Figure $\left.5 \mathrm{C}\right)$.

The current findings provide additional scientific support for using longer infusion times and higher doses of HDMTX in patients with T-ALL (i.e., $5 \mathrm{~g} / \mathrm{m}^{2}$ ), and also for more common subtypes of B-lineage ALL with structural variants (chromosomal translocations) creating either the ETV6-RUNX1 or the TCF3$P B X 1$ gene fusions. Once associated with increased late relapse or poor event-free survival, respectively, these 2 B-lineage ALL 
A

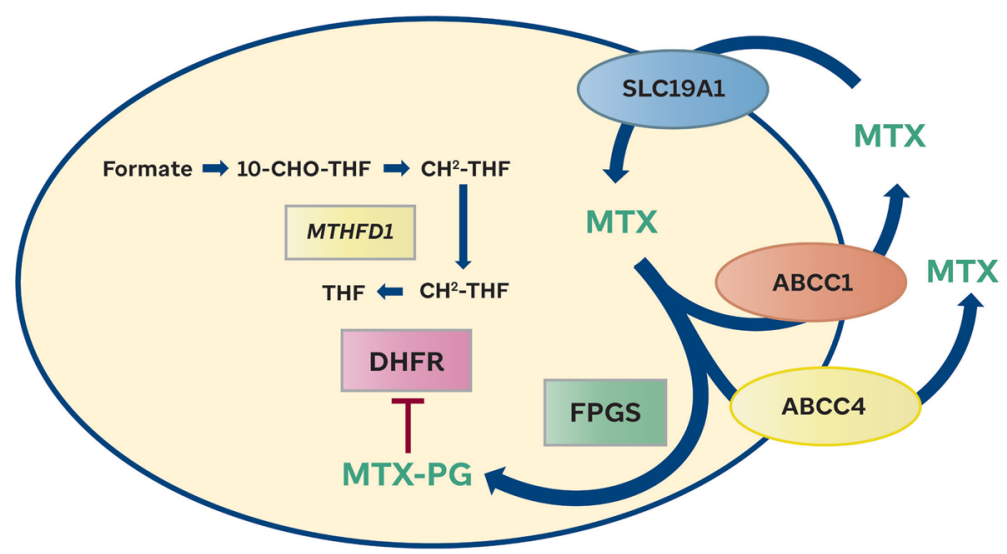

B
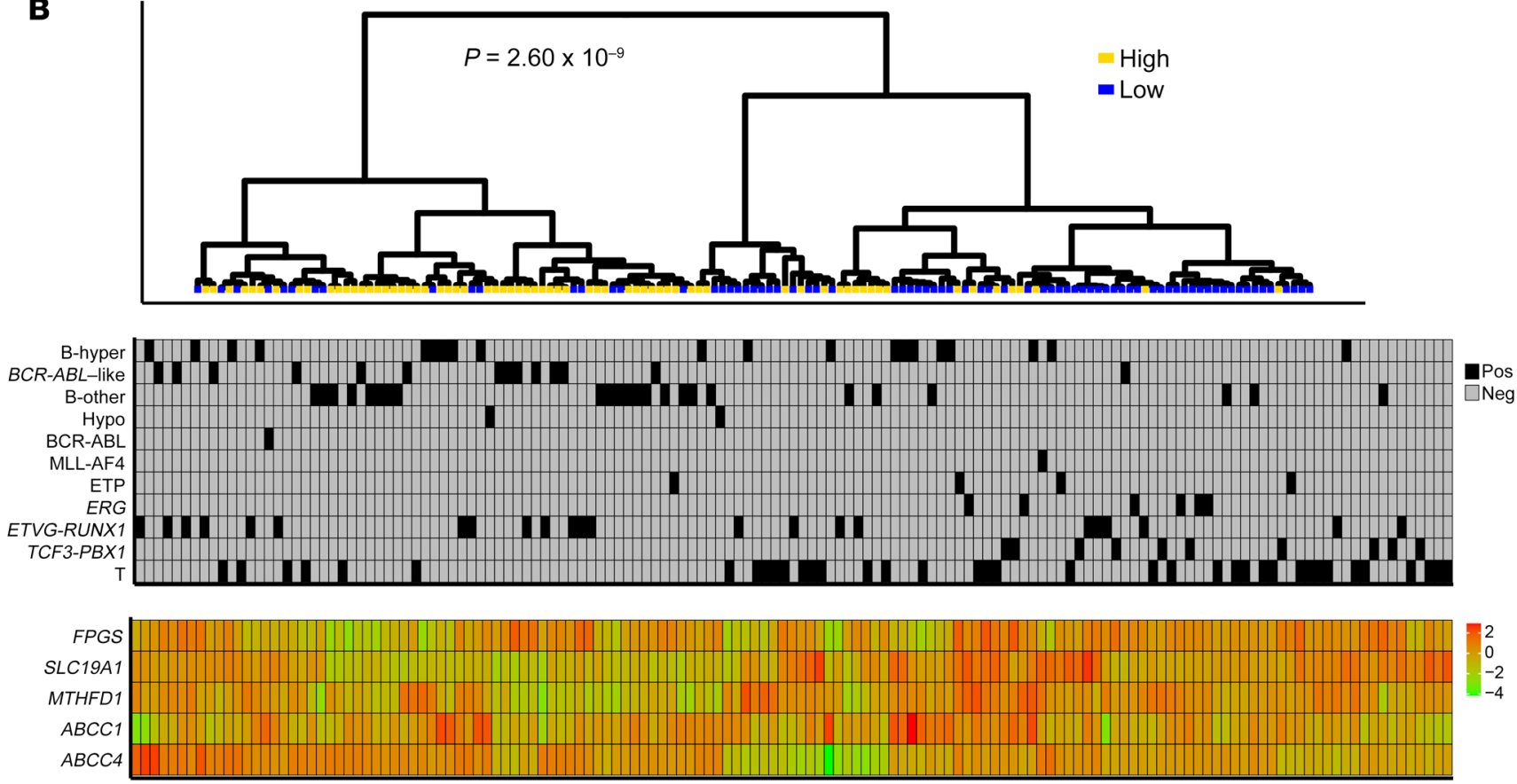

Figure 4. mRNA expression of folate pathway genes that are significantly related to MTXPG accumulation in ALL cells after in vivo HDMTX. (A) Schema of transporters (SLC19A1, ABCC1, ABCC4) and enzymes (FPCS, MTHFD1) involved in folate uptake, efflux, and metabolism that significantly discriminated ALL cells that accumulated high versus low concentrations of MTXPG after in vivo HDMTX treatment. (B) Hierarchical clustering based on mRNA expression of the 5 folate pathway genes that discriminated ALL cells with high (gold) or low (blue) MTXPG accumulation. The clustering $P$ values are shown ( $t$ test). The level of mRNA expression is indicated from low (green) to high (red) according to the scale at the bottom showing the range of log ${ }_{2}$-normalized fluorescence intensity values. Pos, positive; Neg, negative.

subtypes are associated with excellent outcomes in contemporary clinical trials featuring HDMTX treatment (18). These 2 B-lineage ALL subtypes have lower expression of the SLC19A1 gene that encodes the extracellular membrane protein responsible for transporting MTX into leukemia cells. Further, these 2 B-lineage ALL subtypes and the T-lineage ALL subtype have a lower ratio of the MTX influx transporter (SLC19A1) relative to the MTX efflux transporters ( $A B C C 1$ and $A B C C 4)$, consistent with lower net intracellular accumulation of MTX (Figure 5B).

Overexpression of the TCF3-PBX1 fusion gene in human $\mathrm{REH}$ ALL cell lines led to a significant decrease in the expression of SLC19A1 ( 43\%), a decrease in FPGS ( $36 \%)$, and a corresponding decrease in total, long-chain, and short-chain MTXPG after a 24-hour exposure to $1 \mu \mathrm{M}$ MTX (Figure 7), thus recapitulating our in vivo findings in primary ALL cells. Overexpression of TCF3-PBX1 also resulted in a marked increase in the expression of $\mathrm{NOTCH} 2$, a gene that was inversely correlated with the expression of both FPGS and SLC19A1 in primary ALL cells in vivo $\left(P=3.9 \times 10^{-9}\right.$ and $P$ $=5.5 \times 10^{-4}$, respectively). Knockdown $(\sim 80 \%)$ of $N O T C H 2$ in REH or 697 ALL cells led to an approximately $40 \%$ increase in SLC19A1, but no change in FPGS (data not shown), suggesting that the effects of TCF3-PBX1 on SLC19A1 may be via modulation of NOTCH2, whereas its effects on FPGS may be direct, consistent with the strong PBX1-binding site 675 bp upstream of the FPGS TSS (score $604, P<1 \times 10^{-6}$ ). Overexpressing SLC19A1 in the human 697 ALL cell line led to a significant increase in intracellular MTXPG (Fig- 

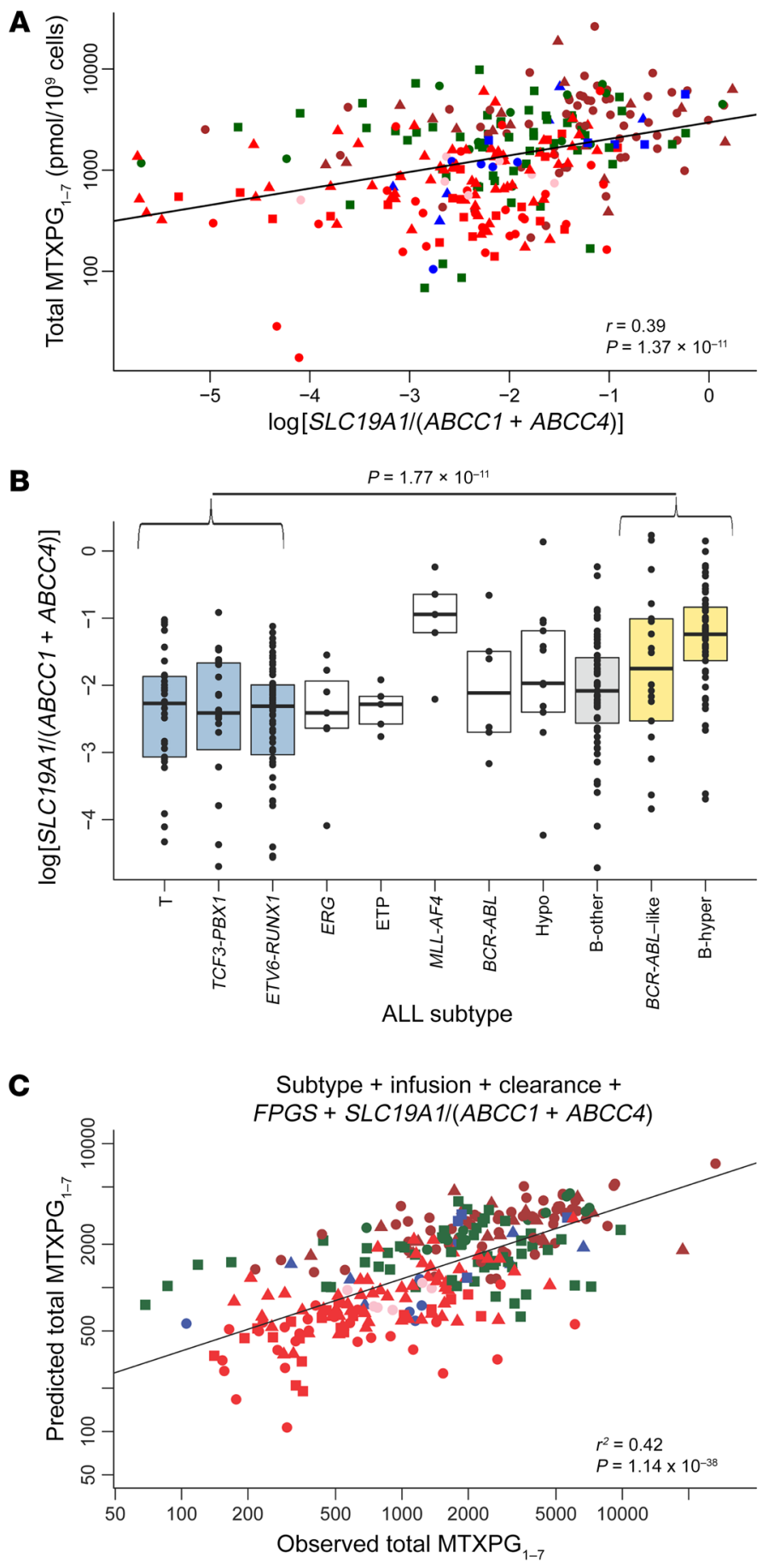

ure 8A), whereas knocking down either SLC19A1 or FPGS led to a significant decrease in intracellular MTXPG in multiple human ALL cell lines (Figure $8 \mathrm{~B})$. These data provide mechanistic insights into why the TCF3-PBX1 ALL subtype has lower MTXPG levels and also support our finding of lower MTXPG accumulation in any patient with ALL who had low expression of SLC19A1 or FPGS.

Our findings have also documented that the more recently discovered and relatively common $B C R-A B L$-like ALL subtype achieves high concentrations of MTXPG, comparable to those seen in hyperdiploid ALL. Both hyperdiploid ALL and the $B C R-A B L$-like subtype express higher levels of SLC19A1 and have a higher folate
Figure 5. Ratio of mRNA expression of the MTX uptake transporter SLC19A1 to expression of the export transporters ABCC1 and ABCC4. (A) Relation of intracellular $\mathrm{MTXPG}_{1-7}$ in primary ALL cells after in vivo treatment with $\mathrm{HDMTX}\left(1 \mathrm{~g} / \mathrm{m}^{2}\right)$ and the ratio of the uptake transporter SLC19A1 to the 2 export transporters $A B C C 1$ and $A B C C 4$ (Pearson's correlation). Each major subtype is depicted with a different symbol, using the same symbols as in Figure 3. (B) ALL subtype differences in the ratio of influx to efflux transporter mRNA expression in primary ALL cells. Blue depicts subtypes with significantly lower MTXPG accumulation versus B-other (in gray) and gold depicts subtype with significant higher MTXPG accumulation. The $P$ value compares the ratio of transporter mRNA in the 3 subtypes in blue and the 2 in gold, indicating a significantly higher influx-to-efflux transporter ratio in ALL with higher MTXPG accumulation ( $P$ value by $t$ test). (C) Relation between $\mathrm{MTXPG}_{1-7}$ concentrations measured in ALL cells after in vivo treatment with HDMTX (observed) versus concentrations predicted by the multivariable model ( $y$ axis). Line depicts the best-fit linear model, linear regression $P=1.14 \times 10^{-38}$ ( $F$ test).

transporter ratio $[S L C 19 A 1 /(A B C C 1+A B C C 4)]$ (Figure 5B). We believe this is the first evidence that $B C R-A B L$-like ALL has favorable accumulation of MTXPG and supports the use of HDMTX as a component of combination chemotherapy for this ALL subtype. Although our study was not designed to address the potential benefits of additional HDMTX in this new higherrisk ALL subtype, our data suggest that this would be worth testing in future clinical trials. Using population model parameters for extracellular and intracellular pharmacokinetics of MTX, we determined by simulation that a 24-hour infusion of approximately $2 \mathrm{~g} / \mathrm{m}^{2}$ HDMTX achieves maximum intracellular MTXPG concentrations in the 2 subtypes with high MTXPG accumulation (i.e., hyperdiploid and $B C R$ - $A B L$-like ALL).

It is noteworthy that, although there was substantial interpatient variation in the expression of dihydrofolate reductase (DHFR) and thymidylate synthase (TYMS) within each ALL subtype and across the entire patient cohort (Supplemental Figure 8), we found no significant difference among the ALL subtypes in the mRNA expression of these MTX target enzymes, except that $D H F R$ expression was significantly higher in TCF3-PBX1 ALL ( $P=0.036 ; \sim 1.4$ times higher than B-other ALL) (Supplemental Figure 8). The levels of mRNA expression of DHFR and TYMS were not significantly related to the leukemia response to HDMTX when ALL subtype and MTXPG levels were covariates in the model. A limitation of our study is the relatively small number of patients within some of the ALL subtype groups.

In summary, we have identified a subtype of ALL (BCR$A B L$-like) that has favorable accumulation of MTXPG after HDMTX treatment, comparable to the good-risk hyperdiploid ALL subtype. We have identified differences in folate pathway genes involved in the metabolism and transport of MTX that provide mechanistic insights into the ALL subtype differences in MTXPG accumulation for this important and widely prescribed antileukemic agent.

\section{Methods}

\section{Patients}

We studied a total of 388 children (aged 18 years or younger) with newly diagnosed ALL who were treated in prospective clinical trials 
Table 2. Folate pathway genes significantly associated with the intracellular accumulation of MTXPG in primary leukemia (ALL) cells, after in vivo treatment with HDMTX $\left(1 \mathrm{~g} / \mathrm{m}^{2}\right)$, based on the $P$ values from the TAP (truncated aggregated $P$ values) statistic

\begin{tabular}{|c|c|c|c|c|c|c|}
\hline Gene & Chrom. & Start & End & TAP $P$ & Obs. TAP stat. & No. of features \\
\hline GART & 21 & 34876238 & 34915797 & $1.7 \times 10^{-10}$ & 136.2 & 45 \\
\hline SHMT1 & 17 & 18231187 & 18266856 & $2.6 \times 10^{-9}$ & 109.1 & 42 \\
\hline SLC19A1 & 21 & 46913486 & 46964325 & $9.1 \times 10^{-9}$ & 73.7 & 34 \\
\hline FPCS & 9 & 130556876 & 130576606 & $1.2 \times 10^{-6}$ & 56.5 & 24 \\
\hline TYMS & 18 & 657604 & 673578 & 0.0024 & 69.9 & 42 \\
\hline MTHFD1 & 14 & 64854749 & 64926722 & 0.0049 & 57.1 & 33 \\
\hline$A B C G 2$ & 4 & 89011416 & 89152474 & 0.0049 & 45.1 & 31 \\
\hline QDPR & 4 & 17461884 & 17513857 & 0.0051 & 53.3 & 54 \\
\hline$A B C C 1$ & 16 & 16043434 & 16236931 & 0.0066 & 71.8 & 81 \\
\hline$A B C C 4$ & 13 & 95672083 & 95953687 & 0.0099 & 119.7 & 139 \\
\hline ATPIF1 & 1 & 28562620 & 28573417 & 0.0408 & 30.1 & 40 \\
\hline$A B C C 2$ & 10 & 101542489 & 101611949 & 0.0428 & 23.2 & 35 \\
\hline
\end{tabular}

After adjusting for multiple testing, $P<0.005$ was considered statistically significant (shown in bold). Chrom., chromosome; Start, start location on the chromosome interrogated; End, end location on the chromosome interrogated; TAP P, TAP statistic; Obs. TAP stat., observed TAP test statistic; No. of features, the number of features interrogated for each gene region.

with HDMTX as an initial single-agent before conventional remission induction chemotherapy at St. Jude Children's Research Hospital (total XIIIA, XIIIB, and XV; NCT00137111; refs. 18-20) and who consented to a bone marrow aspirate for measuring MTXPG in leukemia cells and for genomic studies (Figure 1). ALL cell gene expression and MTXPG were previously reported for 101 of these patients (21); 286 of the patients participated in a randomized study of 4-hour versus 24-hour infusion times for HDMTX (13).

\section{Intracellular MTXPG accumulation}

MTXPGs were measured in primary bone marrow leukemia cells, as previously described $(8,22)$. ALL cells were isolated from bone marrow aspirates obtained 42 to 44 hours after HDMTX treatment, before leucovorin rescue and conventional remission induction therapy.

\section{Pharmacokinetics analysis}

MTX systemic clearance was determined from measured MTX plasma concentrations, as previously described $(22,23)$.

\section{Leukemia subtypes}

Using previously described criteria, each patient's ALL was assigned to 1 of the following 11 subtypes: T-ALL $(n=40)$, early T cell precursor (ETP) $(n=5)$, or B-lineage ALL with either a hyperdiploid karyotype (>50 chromosomes; hyperdiploid, $n=91)$, t $1 ; 19)$ (q23;p13) translocation encoding the TCF3-PBX1 fusion (TCF3-PBX1, $n=24$ ), $\mathrm{t}(12 ; 21)$ (p13;q22) translocation encoding the ETV6-RUNX1 fusion (ETV6-RUNX1, $n=84)$, a BCR-ABL-like $(n=18)$ ALL gene expression pattern with or without $C R L F 2$ rearrangements (IGH-CRLF2; PAR1 deletion or P2RY8-CRLF2) (BCR-ABL-like/CRLF2), t(9;22)

(q34;q11.2) translocation encoding the $B C R-A B L 1$ fusion (BCR$A B L 1, n=9), \mathrm{t}(4 ; 11)(\mathrm{q} 21 ; \mathrm{q} 23)$ translocation encoding the $M L L-A F 4$ fusion ( $M L L-A F 4, n=6$ ), a hypodiploid karyotype with fewer than 44 chromosomes (hypodiploid, $n=16$ ), DUX4/ERG-deregulated ALL ( $n$ $=7$ ), or B-other $(n=88)$, for those patients who had none of these chromosomal aberrations $(12,24,25)$.

\section{Decrease in the leukemia cell count}

Circulating leukemia cells (i.e., WBC) were measured using a Coulter counter (model F_STKR) before therapy $\left(\mathrm{WBC}_{\text {dayo }}\right.$ ) and on day $3\left(\mathrm{WBC}_{\text {day } 3}\right)$ following HDMTX treatment, before the initiation of conventional remission induction chemotherapy. These cell counts were used to assess the in vivo antileukemic effects of

Table 3. Multivariable analysis of FPCS, folate transporter ratio, ALL subtype, MTX systemic clearance and HDMTX infusion time ( $4 \mathrm{~h}$ or $\mathbf{2 4} \mathrm{h}$ ) for their relation to MTXPG accumulation in primary ALL cells, in vivo

B-ALL

Multivariable

Subtype

Transporter ratio

Infusion time

FPCS

MTX clearance

B-ALL, B cell ALL. 

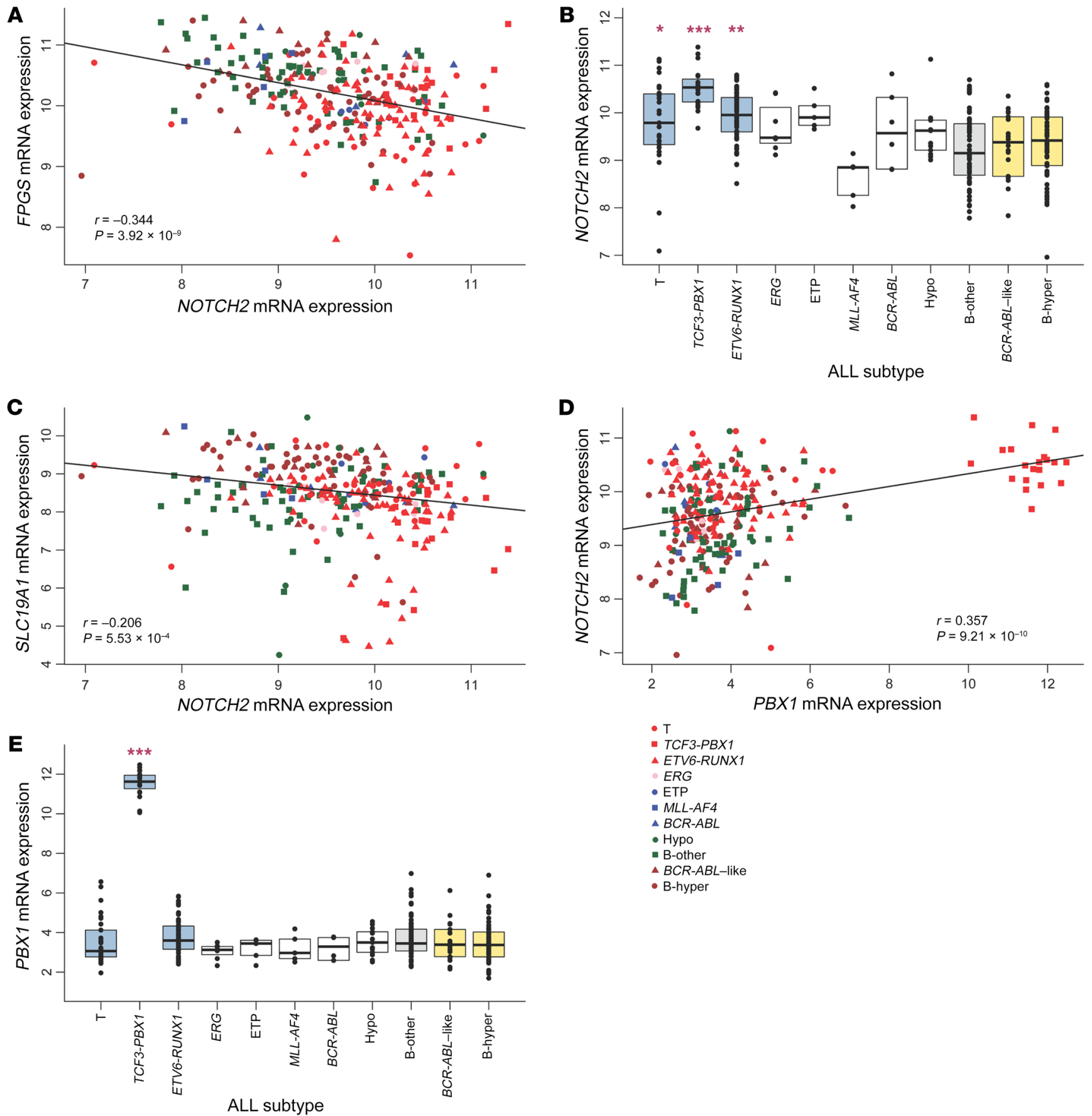

- $\mathrm{T}$

- TCF3-PBX1

$\triangle E T V 6-R \cup N X_{1}$

ERG

- ETP

- MLL-AF4

$\triangle B C R-A B L$

- Hypo

- B-other

$\triangle B C R-A B L-l i k e$

- B-hyper

ALL subtype

Figure 6. Relation between NOTCH2, FPCS, SLC19A1, and PBX1 expression in primary leukemia cells. (A) Relation between NOTCH2 and FPCS mRNA expression in ALL cells. Each ALL subtype is represented by a different symbol, as depicted in Figure 3. (B) ALL subtype differences in NOTCH2 mRNA expression, ordered from left to right for those with the lowest to the highest MTXPG levels (order, blue and gold as defined in Figure 1B). (C) Relation between NOTCH2 and SLC19A1 mRNA expression in ALL cells. Each ALL subtype is represented by a different symbol, as depicted in the symbol key. (D) Relation of NOTCH2 and PBX1 mRNA expression in primary ALL cells. Each ALL subtype is represented by a different symbol, as depicted in the symbol key. (E) ALL subtype differences in $P B X 1$ mRNA expression. Lines depict the best-fit linear model, Pearson's correlation, and $P$ values. ${ }^{*} P<0.05$, ${ }^{* *} P<1 \times$ $10^{-5}$, and ${ }^{* *} P<1 \times 10^{-10}$, by $t$ test.

HDMTX. We used the ALL treatment response phenotype previously described in detail by our group (16), which uses the residuals of the linear regression of log-transformed WBC counts on day 0 and day 3, thus adjusting for the effects of the initial ALL cell count on the overall response metric.

\section{MRD}

MRD was determined by multiparameter flow cytometry, PCR analysis, or both in bone marrow specimens collected on day 19. Either method could routinely detect ALL cells with a sensitivity of $0.01 \%$ or better, and the results were generally concordant. In the 

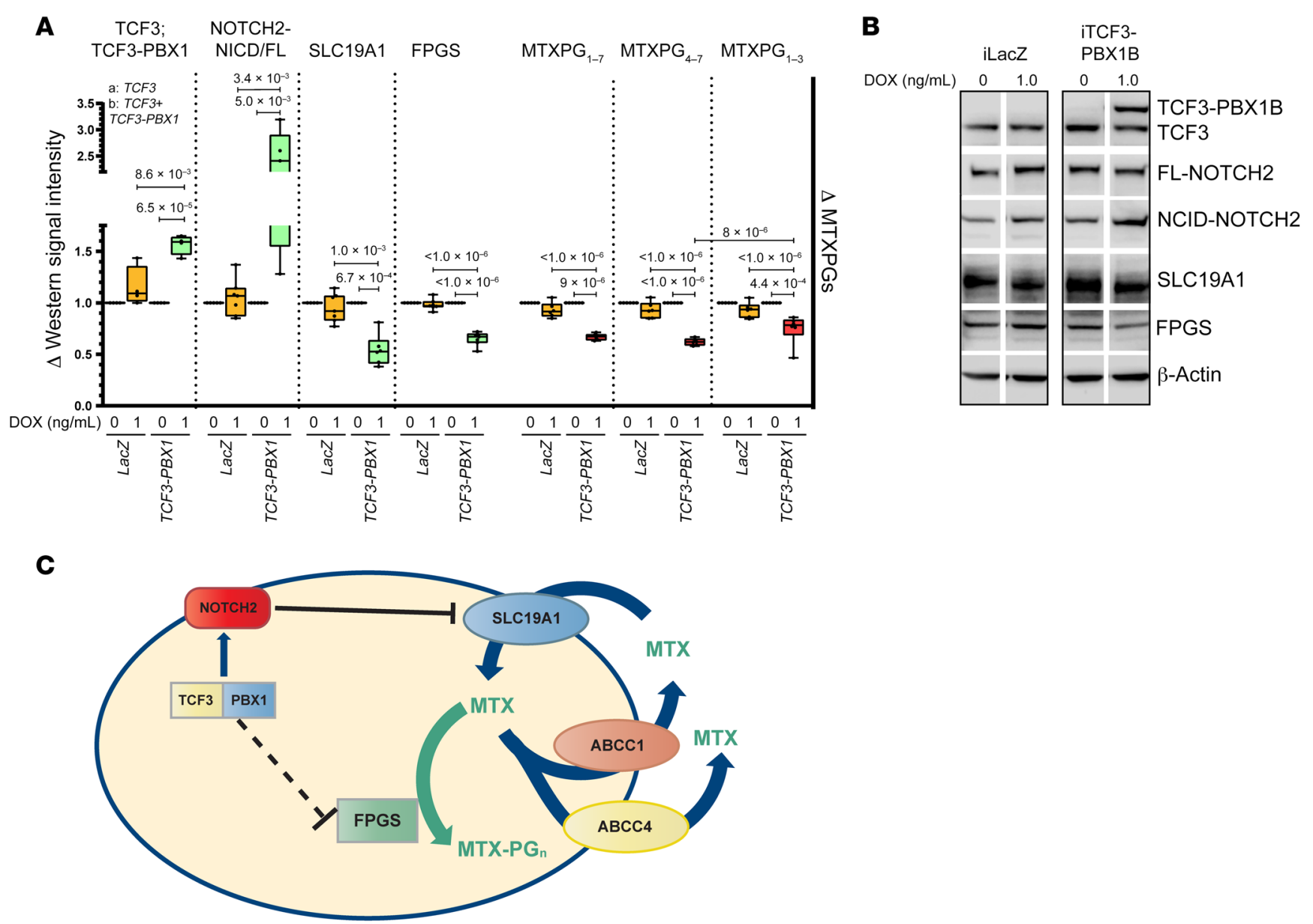

Figure 7. Heterologous overexpression of TCF3-PBX1b in human REH ALL cells modulates NOTCH2, FPCS, and SLC19A1 expression and correlates with decreased MTXPG accumulation. (A and B) Overexpression of the DOX-inducible TCF3-PBX1 protein in the REH human ALL cell line compared with expression in the control LacZ vector. Overexpression of TCF3-PBX1b was associated with a significant increase in expression of NOTCH2-NICD, a significant decrease in expression of SLC19A1 and FPCS, and lower intracellular accumulation of MTXPGs (MTXPG MT $_{17}$, MTPG $_{4-7}, M_{T X P G}$ after incubation with 1 $\mathrm{ng} / \mathrm{mL}$ DOX and $1 \mu \mathrm{M}$ MTX for 24 hours, compared with cells that were not treated with DOX. Each value is the average of 12 samples, compared with the MTXPG concentrations in control cells (value set at 1), expressed as the $\Delta$ increase (values $>1$ ) or decrease (values $<1$ ) compared with controls. $P$ values were calculated by $t$ tests. iLacZ, DOX-inducible LacZ; iTCF3-PBX1B, DOX-inducible TCF3-PBX1B. FL-NOTCH2, full-length NOTCH2. (C) Schema of the hypothetical relation between TCF3-PBX1 expression and NOTCH2, SLC19A1, and FPGS expression.

few cases with discrepant results, we used the highest MRD value for risk assignment.

\section{Gene expression}

Total RNA was extracted and hybridized to the HG-U133A (GPL96) or HG-U133 Plus 2.0 (GPL570) oligonucleotide microarrays (Affymetrix) as previously described (26). Data were deposited in the NCBI's Gene Expression Omnibus (GEO) database (GSE66702; https://www.ncbi. nlm.nih.gov/geo/query/acc.cgi?acc=GSE66702). mRNA expression data were available for 278 patients.

The folate transporter ratio was determined as the ratio of signal intensity of SLC19A1 mRNA relative to the sum of the signal intensities of the $A B C C 1$ and $A B C C 4$ genes.

\section{miRNA expression}

Total RNA was extracted and hybridized to miRCURY LNA 10.0 generated from ready-to-spot probe sets or preprinted fifth generation miRCURY LNA microRNA microarrays (QIAGEN) as previously described
(27). Data were deposited in the NCBI's GEO database (GSE76849; https://www.ncbi.nlm.nih.gov/geo/query/acc.cgi?acc=GSE76849).

\section{DNA methylation}

Genome-wide DNA methylation status was determined as previously described (26) using the Infinium HumanMethylation27 BeadChip or Infinium HumanMethylation450 BeadChip kit (Illumina). Data were deposited in the NCBI's GEO database (GSE67044; https://www. ncbi.nlm.nih.gov/geo/query/acc.cgi?acc=GSE67044 and GSE67043; https://www.ncbi.nlm.nih.gov/geo/query/acc.cgi?acc=GSE67043).

\section{CNAs}

Using the QIAGEN Blood and Cell Culture DNA Kit, DNA was extracted from diagnostic bone marrow samples from 189 patients with sufficient amounts of ALL cell DNA. For each DNA sample, $500 \mathrm{ng}$ DNA was digested with NspI and StyI restriction enzymes, amplified, labeled, and hybridized to the Affymetrix GeneChip Human Mapping $500 \mathrm{~K}$ set. The SNP arrays were processed using the R packages tree 
A

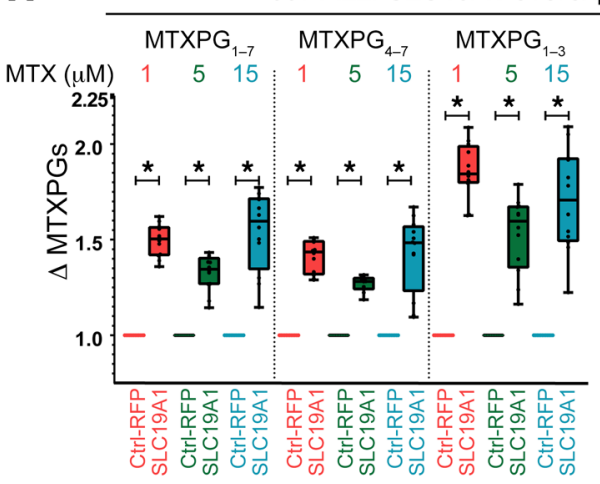

B

SLC19A1-KD
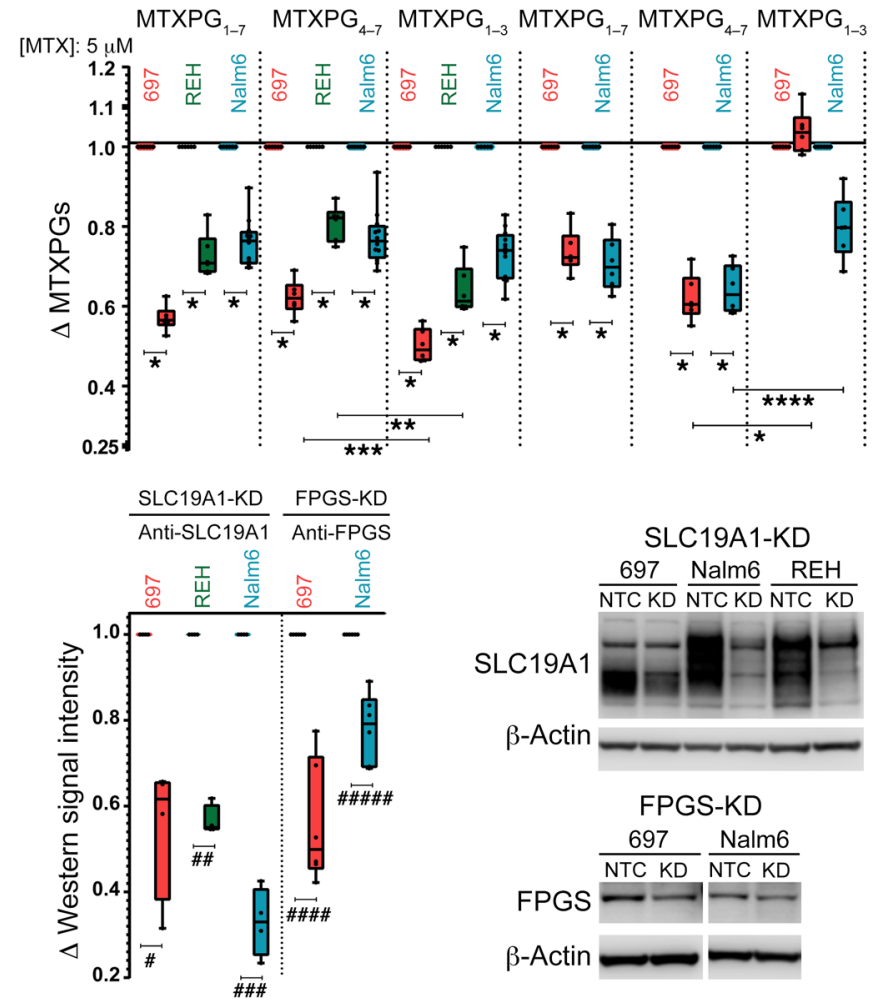

Figure 8. Manipulation of SLC19A1 and FPCS expression in human ALL cell lines and the effects on intracellular MTXPG accumulation. (A) Overexpression of SLC19A1 in human 697 ALL cell line altered intracellular concentrations of total MTXPGs (MTXPG ${ }_{1-7}$ ), long-chain MTXPGs $\left(\mathrm{MTXPG}_{4-7}\right)$, and short-chain MTXPGs (MTXPG ${ }_{1-3}$ ) compared with 697 ALL cells overexpressing RFP cDNA (Ctrl-RFP). The graph shows the quantitation of SLC19A1 protein in the Western blot for control and SLC19A1-overexpressing ALL cells. ${ }^{*} P<1 \times 10^{-6}$; ${ }^{* *} P$ $=2 \times 10^{-3}$. (B) Knockdown (KD) of SLC19A1 or FPGS in human 697, REH, and NALM6 ALL cell lines compared with the NTC. Plot shows intracellular concentrations of total MTXPGs (MTXPG ${ }_{1-7}$ ), long-chain MTXPGs (MTXPG $\left.{ }_{4-7}\right)$, and short-chain MTXPGs (MTXPG ${ }_{1-3}$ ) in ALL cells in which either SLC19A1 or FPGS was knocked down by an shRNA. Significantly lower intracellular total MTXPG accumulation (MTXPG ${ }_{1-7}$ ) was measured in all SLC19A1- and FPGS-knockdown ALL cell lines treated with $5 \mu \mathrm{m}$ MTX for 24 hours compared with NTC ALL cells. In the 3 ALL cell lines with SLC19A1 knockdown, there was a greater decrease in accumulation of short-chain MTXPGs (MTXPG ${ }_{1-3}$ ) than of long-chain MTXPGs (MTXPG ${ }_{4-7}$ ). In contrast, in ALL cells with FPGS knockdown, there was a significantly greater decrease in accumulation of long-chain MTXPGs (MTXPG $\left.{ }_{4-7}\right)$ than of short-chain MTXPGs. Each value represents the average of 12 samples compared with the MTXPG concentrations in control cells (value set at 1 ), expressed as the $\Delta$ increase (values $>1$ ) or decrease (values $<1$ ) compared with controls. $P$ values were determined by $t$ test. The signal intensity plot shows quantitation of the protein signals in the Western blots for shRNA-induced knockdown of SLC19A1 and FPCS. ${ }^{*} P<1 \times 10^{-6} ;{ }^{* *} P<2.0 \times 10^{-5} ;{ }^{* *} P<8.3$ $\times 10^{-4} ;{ }^{* * *} P<4.5 \times 10^{-4} ; \# P=1.4 \times 10^{-3} ; \# \#<1.0 \times 10^{-3}$; \#\#\# $P=$ $3.0 \times 10^{-6} ; \# \# \#=1.9 \times 10^{-5} ; \# \# \# \#=5.9 \times 10^{-5}$. and DNAcopy. First, for each probe set, the raw signal was computed as the mean of the log-transformed Affymetrix CEL file intensities across all probes annotated to that probe set. Next, the raw signals were adjusted for platform and probe type by $Z$ score transformation within each category defined by the combination of these 2 platforms. The $Z$ scores were then scaled by a pooled SD estimate. A regression tree was used to model the distribution of these rescaled $Z$ scores as a function of the guanine-cytosine (GC) content of the probe set oligonucleotide sequence. The regression tree residuals were then further adjusted by subtracting the median, removing first-order autocorrelation of the values ordered by chromosome and position, and rescaling the results by the Rice estimator of the SD based on first differences. These results were then $Z$ transformed within each terminal node of the regression tree to obtain the adjusted signals that were used for change-point detection by circular binary segmentation. The adjusted signals and segment means were then recentered by subtracting the median of the vector that assigned each probe set its segment mean value. Finally, segments with a mean of less than -0.2 were considered to have fewer than 2 copies, and segments with a mean of greater than +0.2 were considered to have more than 2 copies.

\section{Germline SNP genotyping}

Germline DNA was extracted and genotyped using the Affymetrix GeneChip Human Mapping 500K set or the SNP 6.0 array (Affymetrix) as previously described (22). Data were deposited in the NCBI's database of genotypes and phenotypes (dbGaP) (accession number phs000637.v1.p1; https://www.ncbi.nlm.nih.gov/projects/gap/cgibin/study.cgi?study_id=phs000637.v1.p1). SNPs with call rates of less than $95 \%$ in all patients or a minor allele frequency of less than $1 \%$ were filtered out.

\section{Exome-sequencing coding variants}

DNA was extracted from ALL cells isolated from the bone marrow or peripheral blood using the Blood and Cell Culture DNA Kit (QIAGEN). 


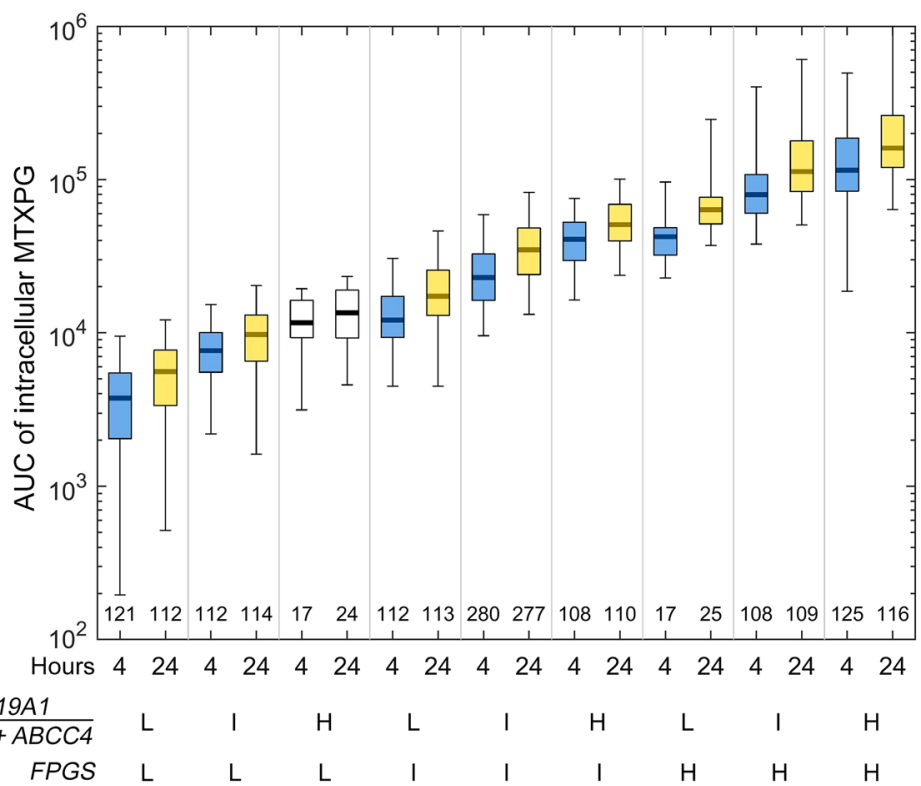

Figure 9. Model-simulated differences in MTXPG accumulation after a 4-hour versus 24-hour i.v. infusion of HDMTX. Model-simulated accumulation of MTXPGs in ALL cells, in vivo, after a 4-hour or 24-hour i.v. infusion of HDMTX $\left(1 \mathrm{~g} / \mathrm{m}^{2}\right)$. A total of 1000 model simulations were performed as described in Methods and depict patients with combinations of a low $(L)$, intermediate $(\mathrm{I})$, or high $(\mathrm{H})$ folate influx/efflux transporter ratio [SLC19A1/(ABCC1 + $A B C C 4$ )] and $F P C S$ expression ( $L$ = first quartile, $\mathrm{I}=$ middle 2 quartiles, and $\mathrm{H}=$ top quartile for efflux/influx transporter ratio and FPCS). The numbers below each box-and-whisker plot indicate the number of patients in that group. Blue IQRs indicate significantly lower MTXPG levels with the 4-hour infusion when compared with the corresponding gold IQR with a 24-hour infusion ( $P<0.05$, Kruskal-Wallis). In simulations in which the difference in MTXPG levels was not statistically significant, the IQRs are shown in white.
Alignment to the reference human genome assembly GRCh37-lite was performed with Burrows-Wheeler Aligner (BWA) and analyzed as previously described using a matched germline sample as a reference for somatic mutations (28). Mutations were filtered for nonsynonymous variants only in the coding region (excluding 3 ' - and 5'-UTR variants). The mutations were then aggregated to individual genes and genelevel clustering via the Ward method using Euclidean distance.

\section{Manipulation of candidate genes in human ALL cell lines}

Leukemia cell lines. Human B-lineage ALL cell lines (REH, NALM6, and 697; DSMZ - German Collection of Microorganisms and Cell Cultures $\mathrm{GmbH}$ ) were maintained in RPMI 1640 supplemented with $10 \%$ FBS (Hyclone Characterized FBS, US origin) and $2 \mathrm{mM} \mathrm{L}$-glutamine (Gibco, Thermo Fisher Scientific) at $37^{\circ} \mathrm{C}$ with $5 \% \mathrm{CO}_{2}$. Stable SLC19A1 knockdown in REH, NALM6, and 697 cells, and FPGS knockdown in NALM6 and 697 cells was achieved by transduction with lentivirus containing an shRNA targeting SLC19A1 (Origene; pGFP-C-shLenti shRNA TL301658C, 5'-TGTCTTCCAAGCGACGGRGRRCAGAATGT-3') or FPGS (Origene; pGFP-C-shLenti shRNA TL312928B, 5'-AGGTGTCATCCACAGGCAACGCAGACCAA-3'). Control REH, NALM6, and 697 cell lines were generated by transduction with nontargeting control (NTC) lentivirus (Origene; pGFP-CshLenti TR30021, scrambled negative control noneffective shRNA). Stably transduced cells were selected by FACS for GFP using a FACSMelody Cell Sorter (BD). A SLC19A1 cDNA fragment (GenBank NM_194255.3) with a GCTAGC KOZAK sequence and 5' NheI and 3' BamHI restriction sites was custom synthesized by GenScript. The NheI/BamHI $1.88 \mathrm{~kb}$ SLC19A1 insert was cloned into the lentivirus expression plasmid pCDH-CMV-MCS-EF1 $\alpha$-RFP+Puro (Systems Biosciences: CD516B-2) linearized with NheI and BamHI. The 697 cells were transduced with SLC19A1-RFP-expressing lentivirus or control RFP-expressing lentivirus, and stably transduced cells were selected by FACS for red fluorescent protein (RFP).

Stable overexpression of SLC19A1 in 697 cells and stable knockdown of SLC19A1 and FPGS in the various ALL cell lines was determined by Western blot analysis using anti-SLC19A1 and anti-FPGS antibodies (see the section Immunoblot detection of TCF3, TCF3$P B X 1 b$, NOTCH2, FPGS, and SLC19A1), and the expression of these 2 proteins was normalized to the expression of $\beta$-actin and to their expression in nontargeting control cells.

Generation of a doxycycline-inducible TCF3-PBX1b-expressing REH ALL cell line. A 2229 bp TCF3-PBX1b cDNA fragment containing a GCCACC KOZAK sequence and 5' EcoRI and 3' PmeI restriction sites was custom synthesized by GenScript. This cDNA fragment contained 1450 bp of the $5^{\prime}$ TCF3 transcript (GenBank NM_003200) and 779 bp of the $3^{\prime}$ end of the PBX1b transcript (GenBank NM_001204961.1). The fusion transcript encodes a 742 amino acid TCF3-PBX1b fusion protein. The TCF3-PBX1b EcoRV/PmeI cDNA insert was cloned into the Plenti-Puro plasmid (Addgene plasmid 39481) DNA linearized with EcoRV/PmeI restriction enzymes. Other lentivirus expression plasmids obtained from Addgene were as follows: pLenti-CMV-TetRBlast (Addgene plasmid 17492) and pLenti-Puro-LacZ (Addgene plasmid 39447). REH cells were first transduced with a pLentiCMV-TetR lentivirus, followed by blasticidin antibiotic resistance selection. Stable TetR expression was verified by Western blotting using a mouse monoclonal TetR antibody (Clontech, 631132). Subsequently, TetR-expressing REH cells were transduced with either the pLenti-Puro-TCF3-PBX1b or Plenti-Puro-LacZ lentivirus, followed by puromycin antibiotic resistance selection. Expression of LacZ or TCF3-PBX1b in the corresponding REH cell lines was induced with doxycycline (DOX) (MilliporeSigma) from $0.20 \mathrm{ng} / \mathrm{mL}$ up to $1 \mu \mathrm{g} / \mathrm{mL}$, and it was determined that $1 \mathrm{ng} / \mathrm{mL}$ DOX resulted in optimal induction of protein expression for 24 hours (data not shown). The experimental results shown in Figure 7A were obtained in the presence or absence of $1 \mathrm{ng} / \mathrm{mL}$ DOX for 24 hours.

Immunoblot detection of TCF3, TCF3-PBX1b, NOTCH2, FPGS, and SLC19A1. Cells were pelleted at 500g. Lysates were prepared in RIPA buffer (MilliporeSigma) containing cOmplete Protease Inhibitor Cocktail (Roche Life Science) and PhosStop phosphatase inhibitor cocktail (MilliporeSigma). Equivalent amounts of protein extracts $(20 \mu \mathrm{g})$ were separated on $4 \%-12 \%$ Novex Bis-Tris polyacrylamide gels (Thermo Fisher Scientific) and transferred onto PVDF (0.45 $\mu \mathrm{m})$ membranes 
(MilliporeSigma). Nonspecific antibody binding was blocked with 5\% nonfat dry milk powder in TBS with 0.05\% Tween-20 (Bio-Rad) for at least 1 hour before incubation of membranes with primary and secondary antibodies. The following primary antibodies were used: rabbit monoclonal anti-E2A (TCF3) (Cell Signaling Technology; D2B1, 12258), which binds to both endogenous TCF3 and the TCF3-PBX1b fusion protein, diluted 1:1000; rabbit polyclonal anti-FPGS (LifeSpan Biosciences; LS-C680494), diluted 1:1000; rabbit polyclonal anti-SLC19A1 (MilliporeSigma; HPA024802), diluted 1:1000; rabbit monoclonal anti-NOTCH2 (Cell Signaling Technology; D76A6, 5732), diluted 1:1000; and mouse monoclonal anti- $\beta$-actin (MilliporeSigma; A5441), diluted 1:100,000. Membranes were then incubated with anti-mouse IgG (Jackson ImmunoResearch; NC9736727) or anti-rabbit IgG (Jackson ImmunoResearch: NC9736726) HRP-conjugated secondary antibodies and developed with SuperSignal West Femto Chemiluminescent Substrate (Thermo Fisher Scientific) before signal acquisition using an Odyssey Fc Imager (LI-COR). Image acquisition, processing, and signal quantification were performed with Image Studio software, version 4.0 (LI-COR). Quantification of delta signal intensities of TCF3-PBX1b, TCF3, NOTCH2, SLC19A1, and FPGS (Figures 7 and 8) was done using the averages of 3 Western blots each. Error bars indicate the SD, and $P$ values were calculated by multiple $t$ tests using GraphPad Prism 8.4 (GraphPad Software).

MTXPG accumulation in cell lines. The various TCF3-PBX1b, FPGS, and SLC19A1 stable expression-manipulated ALL cell lines and matching negative control cell lines were incubated for 24 hours at $37^{\circ} \mathrm{C}$ in complete medium in the presence or absence of $1,5,10$, or $15 \mu \mathrm{M}$ MTX, and $1 \mathrm{ng} / \mathrm{mL}$ DOX in the case of inducible TCF3-PBX1b and LacZ REH cells. Cells were centrifuged at $500 \mathrm{~g}$ for 5 minutes and washed and centrifuged twice in chilled D-PBS (Gibco, Thermo Fisher Scientific). Cell densities and cell viability (trypan blue; MilliporeSigma) were measured using a TC20 Automated Cell Counter (Bio-Rad), and aliquots of $1 \times$ $10^{-6}$ cell pellets were lysed in $100 \mu \mathrm{L}$ ultra-pure water and then frozen at $-80^{\circ} \mathrm{C}$. Each treatment of cell lines and MTX and/or DOX treatment was done in duplicate, and for each experiment, a total of 6 replicates were analyzed to determine the MTXPG concentration. MTX quantification of MTXPG $_{1-7}$ in cell lysates was performed using ultra-high-performance liquid chromatography (UPLC) with mass spectrometric (MS) detection. Mobile phase A was $10 \mathrm{mM}$ ammonium bicarbonate adjusted to $\mathrm{pH} 10$ (790 mg ammonium bicarbonate, $1 \mathrm{~L}$ type 1 water, adjusted with $30 \%$ ammonium hydroxide to $\mathrm{pH} 10$ ), and solvent $\mathrm{B}$ was $\mathrm{MeOH}$ (Thermo Fisher Scientific). MTXPG stock solutions were prepared at $1 \mathrm{mM}$ in 50 $\mu \mathrm{M} \mathrm{NaOH}$ for $\mathrm{MTXPG}_{1}$ and in type 1 water for $\mathrm{MTXPG}_{2 \rightarrow 7}$. Intermediate MTXPG working standards were made in water at 5000,1000, and 100 $\mathrm{nM}$ for $\mathrm{MTXPG}_{1-3}$ and $\mathrm{MTXPG}_{6-7}$ and at 5000, 1000, and $500 \mathrm{nM}$ for MTXPG $_{4-5}$. Six calibration standards were made with 1, 5, 20, 50, 100, and $200 \mathrm{nM} \mathrm{MTXPG}_{1-3}$ and $\mathrm{MTXPG}_{6-7}$ and 6, 10, 20, 50, 100, and 200 nM MTXPG $_{4-5^{\circ}}$ Quality control (QC) standards were made with 5, 30, and $150 \mathrm{nMMTXPG}_{1-3}$ and $\mathrm{MTXPG}_{6-7}$ and 18, 30, and $150 \mathrm{MTXPG}_{4-5}$. Internal standard (IS) stock solutions of $\mathrm{MTXPG}_{1-7}-\mathrm{d} 4$ were prepared in DMSO at $1 \mathrm{mg} / \mathrm{mL}$ and diluted in water to $1.1 \mu \mathrm{M}$ for the working IS solution. A 100 $\mu \mathrm{L}$ aliquot of MTXPG samples, calibration standards, and QC standards were prepared by adding $10 \mu \mathrm{L}$ of the working IS solution into each tube. Samples were heated at $95^{\circ} \mathrm{C}$ for 5 minutes, cooled on ice for 10 minutes, and centrifuged at $21,130 \mathrm{~g}$ for 10 minutes at $4^{\circ} \mathrm{C}$. The supernatant was transferred and injected for ULPC-MS detection. The UPLC parameters were as follows: column (ACQUITY BEH C18 1.7 u 100A; Waters), guard column (ACQUITY in-line filter), mobile phase, column temperature at $40^{\circ} \mathrm{C} \pm 5^{\circ} \mathrm{C}$, autosampler temperature at $10^{\circ} \mathrm{C} \pm 5^{\circ} \mathrm{C}$, flow rate at 0.6 $\mathrm{mL} /$ minute, run time for 12 minutes, preinjection wash at 0 seconds, and postinjection wash at 6 seconds. UPLC gradient program was as follows: $1 \%$ B from 0 to 1 minute; $5 \%$ B at 2.5 minutes; $15 \%$ B at 5 minutes; $30 \%$ B at 5.4 minutes; $95 \%$ B from 5.41 to 8 minutes; and $1 \%$ B from 9 to 12 minutes. MS detection of MTXPG MRM mass transitions $(m / z):$ MTXPG $_{1}$ $455.1 \rightarrow 308.1 ;$ MTXPG $_{2} 584.2 \rightarrow 308.1 ;$ MTXPG $_{3}$ 713.3 $\rightarrow 308.2 ;$ MTXPG $_{4}$ $842.3 \rightarrow 308.2$ MTXPG $_{5} 486.3 \rightarrow 308.2 ;$ MTXPG $_{6} 550.8 \rightarrow 175.0 ;$ MTX$\mathrm{PG}_{7}$ 615.3 $\rightarrow 175.1 ;$ MTXPG $_{1}-\mathrm{d} 3458.2 \rightarrow 311.1 ;$ MTXPG $_{2}-\mathrm{d} 3$ 587.2 $\rightarrow 311.1$; MTXPG $_{3}$-d3 $716.3 \rightarrow 311.1 ; \quad$ MTXPG $_{4}$-d3 $845.3 \rightarrow 311.1 ; \quad$ MTXPG $_{5}$-d3 $487.7 \rightarrow 311.2 ;$ MTXPG $_{6}$-d3 $552.3 \rightarrow 175.1 ;$ MTXPG $_{7}$-d3 $616.8 \rightarrow 311.2$. Data collection was performed using MassLynx V1.40 chromatography/ MS software. Each experiment was performed in duplicate, with 3 samples per condition, each assayed in duplicate; thus, each value represents the average of 12 samples compared with the MTXPG concentrations in control cells (value set at 1 ), expressed as the $\Delta$ increase (values $>1$ ) or decrease (values $<1$ ) compared with controls. Error bars indicate the SD. $P$ values were calculated by multiple $t$ tests using GraphPad Prism 8.4. The MTXPG results are shown in Figures 7 and 8.

\section{Statistics}

Multiple linear regression modeling was used to evaluate the association between MTXPG accumulation (log-transformed) in patients' samples and MTX infusion time, MTX systemic clearance during the treatment window, and ALL subtype, using all available patient data ( $n$ $=388$ ). The differences in MTXPG accumulation among ALL subtypes were evaluated using a 2-tailed $t$ test. Logistic regression analysis was used to examine the relationship between MTXPGs and MRD status, adjusting for leukemia subtypes. Pearson's correlation was used to measure the relationship between MTXPG and antileukemia efficacy of HDMTX as determined on the basis of WBC measurements before and after MTX administration, as previously described (16).

Multiple linear regression was performed to assess the association between MTXPGs and gene expression, with MTX infusion time and MTX plasma clearance as covariates. The folate transporter ratio was analyzed in a manner similar to that for gene expression. A final multivariable linear regression was constructed using the expression of top selected genes, the folate transporter ratio, the leukemia subtype, and the aforementioned covariables. The correlation between the expression of individual genes and other genes or MTXPGs was depicted using Pearson's correlation. Hierarchical clustering was performed to cluster patients with top/bottom quartiles of MTXPG using selected gene expression probe sets. The MTXPG levels of the patients in the top 2 major clusters were compared using a 2 -tailed $t$ test. The gene expression differences among ALL subtypes were evaluated using a 2-tailed $t$ test. Bonferroni's $P$ value threshold was applied in the screening of top folate genes on the basis of gene expression to account for multiple testing.

A $P$ value of less than 0.05 was considered significant unless otherwise specified. All statistical analyses were performed using the statistical environment R 3.6.1 (R Development Core Team, http:// www.r-project.org)

\section{Integrated gene level (TAP) analysis}

To assess a known gene's potential involvement in leukemia cell in vivo accumulation of MTXPG, we combined evidence from all 6 genomic and epigenomic features (mRNA expression, methylation, miRNA 
expression, leukemia cell CNAs, somatic mutations by WES, and genotype of the inherited SNPs) in proximity of $(50 \mathrm{~kb})$ all 47 folate pathway genes. Each feature was evaluated individually for its association with MTXPG as described previously, and the significant features (linear regression $P<0.05$ ) were included in the overall gene-level model. A hybrid permutation approach was used along with a nonparametric smooth cumulative distribution function $(\mathrm{CDF})$ with a variationdiminishing spline to obtain a TAP statistic for every gene $(29,30)$. Adaptive thresholding was used, as previously described, to define the threshold of significance (31). Custom-generated R code was used to perform the TAP analysis as previously described (28) can be found on GitHub https:/github.com/evanslabSJCRH/PolygenomicAnalysis/tree/master/TAP; GitHub commit ID: b46b652).

\section{Model simulations of intracellular MTXPG}

We used a pharmacokinetic model of extracellular MTX and intracellular MTXPG that we have previously described in detail (32). The model consists of 2 components. First, extracellular plasma MTX is described with a linear first-order, 2-compartment pharmacokinetic model (Equation 1) with the parameters: $\mathrm{k}_{\mathrm{e}}(1 / \mathrm{h})$, elimination of plasma MTX; V ( $\left.\mathrm{L} / \mathrm{m}^{2}\right)$, systemic volume; and, $\mathrm{k}_{12}$ and $\mathrm{k}_{21}(1 / \mathrm{h})$, intercompartmental parameters.

$$
\begin{aligned}
& \frac{d M T X}{d t}=-\left(k_{e}+k_{12}\right) M T X+k_{21} M_{T} X_{p} \\
& \frac{d M T X_{p}}{d t}=k_{12} M T X-k_{21} M T X_{p}
\end{aligned}
$$

(Equation 1)

Second, intracellular MTXPG disposition was described with 2 compartments (Equation 2) accounting for intracellular MTX or MTX$\mathrm{PG}_{1}$ and the sum of MTXPG $\mathrm{M}_{2}$ through $\mathrm{MTXPG}_{7}$ or $\mathrm{MTXPG}_{2-7}$ (the subscript on MTXPG denotes the number of glutamates attached to the MTX molecule). Specifically, active influx of MTX into leukemic blasts (e.g., the reduced folate carrier, encoded by SLC19A1) was described by Michaelis-Menten kinetics with parameters $\mathrm{V}_{\text {max-influx }}\left(\mathrm{pmol} / 10^{9}\right.$ cells $/ \mathrm{h}$ ) and $\mathrm{K}_{\mathrm{m} \text {-influx }}(\mu \mathrm{M})$. Passive influx was also described with the first-order kinetic parameter $\mathrm{k}_{\text {influx }}(1 / \mathrm{h})$. Efflux of MTX (e.g., $A B C C 1$ and $A B C C 4$ ) was described by first-order kinetics with the parameter $\mathrm{k}_{\text {efflux }}(1 / \mathrm{h})$. Formation of polyglutamates (MTXPGs) via FPGS was described by Michaelis-Menten kinetics with the parameters $\mathrm{V}_{\text {max-FPGS }}$ $\left(\mathrm{pmol} / 10^{9}\right.$ cells $/ \mathrm{h}$ ) and $\mathrm{K}_{\mathrm{m}-\mathrm{FPGS}}(\mu \mathrm{M})$. Finally, the cleavage of polyglutamates from MTXPG by $\gamma$-glutamyl hydrolase (GGH) was described by first-order kinetics with the parameter $\mathrm{k}_{\mathrm{GGH}}(1 / \mathrm{h})$.

$$
\begin{aligned}
\frac{M T X P G_{1}}{d t} & =\frac{V_{\text {max-influx }} M T X / V}{V_{m \text {-influx }}+M T X / V}+k_{\text {influx }} M T X / V-k_{\text {efflux }} M T X P G_{1} \\
& -\frac{V_{\text {max-FPGS }} M T X P G_{1}}{K_{m-F P G S}+M T X P G_{1}}+k_{G G H} M T X P G_{2-7} \\
\frac{d M T X P G_{2-7}}{d t} & =\frac{V_{\text {max-FPGS }} M T X P G_{1}}{K_{m-F P G S}+M T X P G_{1}}-K_{G G H} M T X P G_{2-7}
\end{aligned}
$$

(Equation 2)

The MTXPG modeling consisted of sets of 11 model-estimated parameters $\left(\mathrm{k}_{\mathrm{e}}, \mathrm{V}, \mathrm{k}_{12}, \mathrm{k}_{21}, \mathrm{~V}_{\text {max-influx }}, \mathrm{K}_{\mathrm{m} \text {-influx }}, \mathrm{k}_{\text {influx }}, \mathrm{k}_{\text {efflux }}, \mathrm{V}_{\text {max-FPGS }}, \mathrm{K}_{\mathrm{m}-\mathrm{FPGS}}, \mathrm{k}_{\mathrm{GGH}}\right)$ for each individual. Overall, in the initial model development, there were parameter sets for 99 individuals randomized to receive $1 \mathrm{~g} / \mathrm{m}^{2}$ MTX infused i.v. over 24 hours and for 95 individuals randomized to receive $1 \mathrm{~g} / \mathrm{m}^{2}$ MTX infused i.v. over 4 hours. These sets of estimated parameters were used in the current study to simulate the intracellular MTXPG $_{2-7}$ AUC over a 42-hour period. Specifically, 1000 model parameter sets for each infusion length were randomly generated on the basis of the multivariate log-normal distribution of the individual parameter sets using the MATLAB function "mvnrnd" (MATLAB 2019a, MathWorks). Individual MTXPG exposures including the AUC and the maximum intracellular MTXPG concentration (Cmax) were calculated on the basis of these simulated parameters and $1 \mathrm{~g} / \mathrm{m}^{2}$ MTX infused i.v. over 4 or 24 hours.

Individual simulations with model parameters describing the MTX influx to efflux ratio $\left(\mathrm{V}_{\text {max-influx }} / \mathrm{K}_{\text {effux }}\right)$, or formation of intracellular polyglutamates $\left(\mathrm{V}_{\text {max-FPSS }}\right)$ that fell into the lower, middle, and upper quartiles (Q1: <25th percentile, Q23: 25th-75th percentile, and Q4: >75th percentile, respectively) were used to describe simulated individuals with reduced, intermediate, or high MTX net transporter activity [e.g., SLC19A1/(ABCC1 + ABCC4) mRNA expression ratio] or MTXPG formation (e.g., FPGS expression), respectively.

\section{Study approval}

The IRB of St. Jude Children's Research Hospital approved this study, and written informed consent was obtained from patients and/or their parents or legal guardians before enrollment, as appropriate, in accordance with the Declaration of Helsinki.

\section{Author contributions}

CHP, MVR, and WEE designed the clinical trials. JCP conducted the model simulations. BS performed the MTXPG analyses. CC, DP, and LS performed the statistical and TAP analyses. ELL, SWP, JCP, KRC, EJB, CC, DP, WY, RJA, JRM, BD, KGR, LS, SP, CGM, CHP, MVR, CS, and WEE acquired, analyzed, and/or interpreted data. ELL, RJA, CC, and WEE wrote the manuscript (with review and input by all authors).

\section{Acknowledgments}

We gratefully acknowledge the patients and parents who participated in this research. We are appreciative of the expert technical assistance of Yaqin Chu, Emily Melton, Yan Wang, Margaret Needham, Natasha Atkinson, Hannah Williams, Granger Ridout, Deanna Naeve, John Stukenborg, and Jay Morris. This work was supported in part by the NIH NCI grant R37CA36401 (to WEE, MVR, and CHP); the NIH National Institute of General Medical Sciences Pharmacogenomics Research Network grant U01 GM115279 (to MVR and WEE); NIH grants F32CA141762 (to SWP) and R01CA78224 (to WEE); and a grant from the American Recovery and Reinvestment Act supplemental (3R37CA03640126S1, to WEE). ELL was supported by a postdoctoral grant from the Basque Government (Programa Posdoctoral de Perfeccionamiento de Personal Investigador doctor, Departamento de Educación, Política Lingüística y Cultura del Gobierno Vasco) and by grants from the Basque Government (IT989-16) and the Fundación Mutua Madrileña (AP171202019). This work was also supported by NCI Cancer Center Support grant CA 21765 and by the ALSAC. The funders had no role in study design, data collection 
and analysis, decision to publish, or preparation of the manuscript. The content is solely the responsibility of the authors and does not necessarily represent the official views of the NIH.

Address correspondence to: William E. Evans, St. Jude Children's Research Hospital, 262 Danny Thomas Place, Memphis, Tennes- see 38105, USA. Phone: 901.595.6850; Email: william.evans@ stjude.org.

ELL's present address is: Department of Genetics, Physical Anthropology and Animal Physiology, Faculty of Medicine and Nursing, University of the Basque Country, UPV/EHU, Leioa, Spain.
1. Allemani C, et al. Global surveillance of trends in cancer survival 2000-14 (CONCORD-3): analysis of individual records for 37,513,025 patients diagnosed with one of 18 cancers from 322 population-based registries in 71 countries. Lancet. 2018;391(10125):1023-1075.

2. Bassan R, Bourquin JP, DeAngelo DJ, Chiaretti S. New approaches to the management of adult acute lymphoblastic leukemia [published online September 21, 2018].J Clin Oncol. https://doi. org/10.1200/JCO.2017.77.3648.

3. Pui CH, et al. Childhood acute lymphoblastic leukemia: progress through collaboration. JClin Oncol. 2015;33(27):2938-2948.

4. Marin JJ, Monte MJ, Blazquez AG, Macias RI, Serrano MA, Briz O. The role of reduced intracellular concentrations of active drugs in the lack of response to anticancer chemotherapy. Acta Pharmacol Sin. 2014;35(1):1-10.

5. Evans WE, et al. Clinical pharmacodynamics of high-dose methotrexate in acute lymphocytic leukemia. Identification of a relation between concentration and effect. $N$ EnglJMed. 1986;314(8):471-477.

6. Evans WE, Relling MV, Rodman JH, Crom WR, Boyett JM, Pui CH. Conventional compared with individualized chemotherapy for childhood acute lymphoblastic leukemia. $N$ Engl J Med. 1998;338(8):499-505.

7. Synold TW, et al. Blast cell methotrexate-polyglutamate accumulation in vivo differs by lineage, ploidy, and methotrexate dose in acute lymphoblastic leukemia.JClin Invest. 1994;94(5):1996-2001.

8. Masson E, et al. Accumulation of methotrexate polyglutamates in lymphoblasts is a determinant of antileukemic effects in vivo. A rationale for high-dose methotrexate. JClin Invest. 1996;97(1):73-80.

9. Whitehead VM, et al. Accumulation of high levels of methotrexate polyglutamates in lymphoblasts from children with hyperdiploid (greater than 50 chromosomes) B-lineage acute lymphoblastic leukemia: a Pediatric Oncology Group study. Blood. 1992;80(5):1316-1323.
10. Mullighan CG, et al. Deletion of IKZF1 and prognosis in acute lymphoblastic leukemia. $N$ Engl J Med. 2009;360(5):470-480.

11. Den Boer ML, et al. A subtype of childhood acute lymphoblastic leukaemia with poor treatment outcome: a genome-wide classification study. Lancet Oncol. 2009;10(2):125-134.

12. Zhang J, et al. Deregulation of DUX4 and ERG in acute lymphoblastic leukemia. Nat Genet. 2016;48(12):1481-1489.

13. Mikkelsen TS, et al. Shortening infusion time for high-dose methotrexate alters antileukemic effects: a randomized prospective clinical trial. JClin Oncol. 2011;29(13):1771-1778.

14. McIntyre B, Asahara T, Alev C. Overview of basic mechanisms of Notch signaling in development and disease. Adv Exp Med Biol. 2020;1227:9-27.

15. Fornes O, et al. JASPAR 2020: update of the open-access database of transcription factor binding profiles. Nucleic Acids Res. 2020;48(D1):D87-D92.

16. Sorich MJ, et al. In vivo response to methotrexate forecasts outcome of acute lymphoblastic leuke$\mathrm{mia}$ and has a distinct gene expression profile. PLoS Med. 2008;5(4):e83.

17. Jolivet J, Cowan KH, Curt GA, Clendeninn NJ, Chabner BA. The pharmacology and clinical use of methotrexate. N EnglJ Med. 1983;309(18):1094-1104.

18. Pui $\mathrm{CH}$, et al. Treating childhood acute lymphoblastic leukemia without cranial irradiation. N Engl J Med. 2009;360(26):2730-2741.

19. Pui $\mathrm{CH}$, et al. Early intensification of intrathecal chemotherapy virtually eliminates central nervous system relapse in children with acute lymphoblastic leukemia. Blood.1998;92(2):411-415.

20. Pui $\mathrm{CH}$, et al. Improved outcome for children with acute lymphoblastic leukemia: results of Total Therapy Study XIIIB at St Jude Children's Research Hospital. Blood.2004;104(9):2690-2696.

21. Kager $\mathrm{L}$, et al. Folate pathway gene expression differs in subtypes of acute lymphoblastic leukemia and influences methotrexate pharmacodynamics. JClin Invest. 2005;115(1):110-117.
22. French D, et al. Acquired variation outweighs inherited variation in whole genome analysis of methotrexate polyglutamate accumulation in leukemia. Blood. 2009;113(19):4512-4520.

23. Panetta JC, et al. A mathematical model of in vivo methotrexate accumulation in acute lymphoblastic leukemia. Cancer Chemother Pharmacol. 2002;50(5):419-428.

24. Roberts KG, Mullighan CG. Genomics in acute lymphoblastic leukaemia: insights and treatment implications. Nat Rev Clin Oncol. 2015;12(6):344-357.

25. Roberts KG, et al. Targetable kinase-activating lesions in Ph-like acute lymphoblastic leukemia. N EnglJMed. 2014;371(11):1005-1015.

26. Paugh SW, et al. NALP3 inflammasome upregulation and CASP1 cleavage of the glucocorticoid receptor cause glucocorticoid resistance in leukemia cells. Nat Genet. 2015;47(6):607-614.

27. Paugh SW, et al. MicroRNAs form triplexes with double stranded DNA at sequence-specific binding sites; a eukaryotic mechanism via which microRNAs could directly alter gene expression. PLoS Comput Biol. 2016;12(2):e1004744.

28. Autry RJ, et al. Integrative genomic analyses reveal mechanisms of glucocorticoid resistance in acute lymphoblastic leukemia. Nat Cancer. 2020;1(3):329-344.

29. Cheng C, Parzen E. Unified estimators of smooth quantile and quantile density functions. J Stat Plan Infer. 1997;59(2):291-307.

30. DeVore RA. The Approximation of Continuous Functions by Positive Linear Operators. SpringerVerlag; 1972.

31. Cheng C. An adaptive significance threshold criterion for massive multiple hypotheses testing. In: Rojo J, ed. Optimality. Institute of Mathematical Statistics; 2006:51-76.

32. Panetta JC, Sparreboom A, Pui CH, Relling MV, Evans WE. Modeling mechanisms of in vivo variability in methotrexate accumulation and folate pathway inhibition in acute lymphoblastic leukemia cells. PLoS Comput Biol. 2010;6(12):e1001019. 